\title{
ON WEAK PLANE COUETTE AND POISEUILLE FLOWS OF RIGID ROD AND PLATELET ENSEMBLES*
}

\author{
ZHENLU CUI ${ }^{\dagger}$, M. GREGORY FOREST ${ }^{\ddagger}$, QI WANG ${ }^{\S}$, AND HONG ZHOU
}

\begin{abstract}
Films and molds of nematic polymer materials are notorious for heterogeneity in the orientational distribution of the rigid rod or platelet macromolecules. Predictive tools for structure length scales generated by shear-dominated processing are vitally important: both during processing because of flow feedback phenomena such as shear thinning or thickening, and postprocessing since gradients in the rod or platelet ensemble translate to nonuniform composite properties and to residual stresses in the material. These issues motivate our analysis of two prototypes for planar shear processing: drag-driven Couette and pressure-driven Poiseuille flows. Hydrodynamic theories for high aspect ratio rod and platelet macromolecules in viscous solvents are well developed, which we apply in this paper to model the coupling between short-range excluded volume interactions, anisotropic distortional elasticity (unequal elasticity constants), wall anchoring conditions, and hydrodynamics. The goal of this paper is to generalize scaling properties of steady flow molecular structures in slow Couette flows with equal elasticity constants [M. G. Forest et al., J. Rheol., 48 (2004), pp. 175-192] in several ways: to contrast isotropic and anisotropic elasticity; to compare Couette versus Poiseuille flow; and to consider dynamics and stability of these steady states within the asymptotic model equations.
\end{abstract}

Key words. liquid crystals, nematic polymers, asymptotic expansions, partial differential equations, instability

AMS subject classifications. $76 \mathrm{~A} 15,82 \mathrm{D} 60$

DOI. $10.1137 / 04061934 \mathrm{x}$

1. Introduction. Shear dominated flows of nematic liquid crystal polymers (NLCPs) generate anisotropy and spatial heterogeneity in the orientational distribution of the rigid rod or platelet ensemble. These phenomena are well documented in light scattering textures [9, 1, 24, 25, 31]. A characterization of the lengthscales in the molecular distribution responsible for the scattering patterns, and whether they are due to changes in the direction of peak orientation (nematic elasticity) or due to focusing and defocusing of the orientational distribution (molecular elasticity), are the subject of numerous modeling and computational studies (cf. [32, 30, 21, 29]). Molecular orientation features in different flow regimes are of extreme importance for materials design, as they impart anisotropic and nonuniform material properties $[34,18,19]$. Another issue typical of non-Newtonian fluids is flow feedback, where

*Received by the editors November 21, 2004; accepted (in revised form) August 16, 2005; published electronically March 31, 2006. This research was sponsored by Air Force Office of Scientific Research, Air Force Materials Command, grants F49620-02-1-0086 and F49620-03-1-0098; National Science Foundation grants DMS-0204243 and DMS-0308019; and the Army Research Office, Materials Division.

http://www.siam.org/journals/siap/66-4/61934.html

$\dagger$ Department of Mathematics, University of North Carolina at Chapel Hill, Chapel Hill, NC 27599-3250 (zcui@email.unc.edu).

${ }^{\ddagger}$ Department of Mathematics; Institute for Advanced Materials, Nanoscience \& Technology, University of North Carolina at Chapel Hill, Chapel Hill, NC 27599-3250 (forest@amath.unc.edu). The research of this author was supported in part by NASA University Research, Engineering and Technology Institute on Bio Inspired Materials (BIMat) award NCC-1-02037.

$\S$ Department of Mathematics, Florida State University, Tallahassee, FL 32306-4510 and Nankai University, Tianjin, 300071, People's Republic of China (wang@math.fsu.edu).

`Department of Applied Mathematics, Naval Postgraduate School, Monterey, CA 93943 (hzhou@ nps.navy.mil). 
elastic stresses alter apparent viscosity. This paper is a continuation of our systematic studies of mesostructures, both from free space elasticity patterns absent of external fields and boundary anchoring conditions [11, 12], and from planar Couette cells moving at prescribed slow speeds $[13,14]$. We defer to these articles, where a detailed account of analytical results on continuum Leslie-Ericksen-Frank (LEF) models is given, notably by $[26,4,5,7,27,23,28]$.

In [14], the authors considered a Doi-Marrucci-Greco (DMG) mesoscopic orientation tensor model, allowing a full coupling between flow structure, director (nematic) and order parameter (molecular) distortions, and with imposed plate motion and molecular anchoring conditions. The model has been benchmarked in the longwave, monodomain regime with resolved simulations of the Doi kinetic theory $[15,16]$. Indeed, the motivation for an analytical study of structure properties is to provide guidance for structure simulations of mesoscopic [17] and kinetic $[15,16]$ models, where the parameter space is too large to assimilate any kind of collapse of the numerical data through scaling laws.

In this paper, we extend our previous asymptotic scaling analysis in several ways. First, we consider a more general physical model to admit anisotropic distortional elasticity (unequal bend, splay, twist elasticity constants). The second-moment orientation model is derived from a recent generalization of the Doi-Hess-Marrucci-Greco kinetic theory [33] and guides our numerical studies [15, 16], for which there are no preceding numerical or analytical results. Second, the asymptotic analysis is extended from plate-driven Couette cell properties to pressure-driven Poiseuille flows. The boundary conditions consist of molecular orientational anchoring conditions at solid walls, where the degree of order is set by the concentration of the nematic liquid and the principal orientation axis is a free parameter, together with no-slip conditions for the velocity field. We further assume an in-plane orientation tensor (restricting the principal orientation axes of the molecular distribution to the flow-flow gradient plane), and posit that the velocity field varies only transverse to the primary flow direction. These assumptions are not easily lifted, in that the fortuitous diagonalization of the flow-nematic steady balance equations is apparently lost for higher dimensional orientational and spatial degrees of freedom. Finally, we extend the asymptotic analysis to time-dependent model equations.

From this formulation, we develop a formal asymptotic analysis in the slow-plate (so-called small Deborah number) and weak pressure gradient limits, which yield exactly solvable, steady flow-nematic model equations. From the explicit solutions, lengthscale selection criteria and scaling properties become explicit, parameterized in terms of molecular parameters (nematic concentration $N$, molecule aspect ratio $r$, persistence length $\mathcal{L}$ of distortional elasticity, persistence length $L$ of the anisotropic distortional elasticity) [33], and experimental conditions (gap width (2h), plate speeds $\pm v_{0}$ for plane Couette flows and pressure gradient $\frac{\partial p}{\partial x}$ for the plane Poiseuille flow, and plate anchoring conditions on the molecular field). From the time-dependence in the asymptotic equations, we explore transient solutions at the first and second order in the asymptotic scheme to infer stability of the steady states within the asymptotic balance equations. We first consider plane Couette flow, followed in the next section by plane Poiseuille flow.

2. Spatial structures and their stability in plane Couette flows. We consider plane Couette flow between two parallel plates located at $y= \pm h$ and moving with velocity $\mathbf{v}=\left( \pm v_{0}, 0,0\right)$, respectively, in Cartesian coordinates $(x, y, z)$. Figure 1 depicts the cross section of the flow geometry on the $(x, y)$ plane.

Here we consider flow-orientation interactions in weak plane Couette flow, char- 


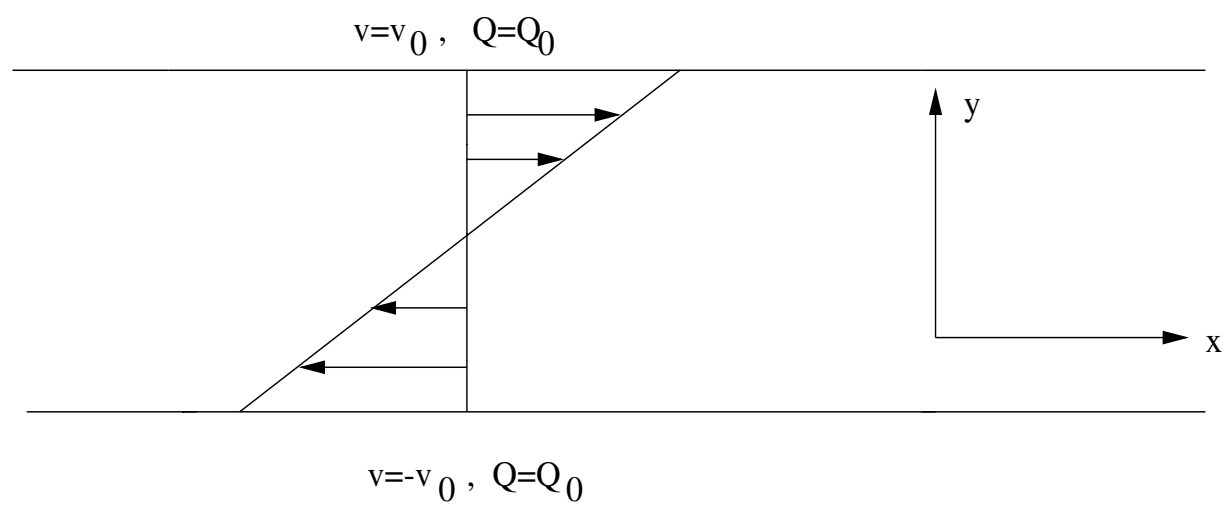

FIG. 1. Geometry of plane Couette flow. The gap width in the shear cell is $2 h$. The LCP in the cell is sheared by moving the upper plate with a constant speed $v_{0}$ and the lower one with the same speed in the opposite direction. At the bounding surfaces, the orientation tensor is equal to its equilibrium value.

acterized by a small effective or averaged shear rate. We nondimensionalize using the gap half-width $(h)$ between the shearing plates and the nematic polymer mean relaxation time $t_{0}=\frac{1}{D_{r}^{0}}$, where $D_{r}^{0}$ is the rotary diffusivity for the rigid rod or platelet [33]. We denote the position vector by $\mathbf{x}$, the velocity by $\mathbf{v}$, the extra stress tensor by $\tau$, and the pressure by $p$, respectively. The dimensionless flow and stress variables are defined by:

$$
\tilde{\mathbf{v}}=\frac{t_{0}}{h} \mathbf{v}, \quad \tilde{\mathbf{x}}=\frac{1}{h} \mathbf{x}, \quad \tilde{t}=\frac{t}{t_{0}}, \quad \tilde{\tau}=\frac{h^{2}}{f_{0}} \tau, \quad \tilde{p}=\frac{h^{2}}{f_{0}} p,
$$

where $f_{0}=\rho h^{4} / t_{0}^{2}$ is a mesophase bulk force and $\rho$ is the nematic polymer (NLCP) density. Let $c$ be the NLCP number density, $k$ the Boltzmann constant, $T$ absolute temperature, $N$ a dimensionless concentration, $\eta_{s}$ the solvent viscosity, and $\zeta_{i}, i=$ $1,2,3$ three friction coefficients related to NLCP-solvent interactions. $\mathcal{L}$ measures the range of isotropic elastic interaction while $L$ does so for the anisotropic elastic interaction [33]. The following eight dimensionless parameters arise:

(2) $R e=\frac{\rho h^{2}}{t_{0} \eta_{s}}, \quad \alpha=\frac{3 c k T t_{0}^{2}}{h^{2} \rho}, \quad E r=\frac{8 h^{2}}{N \mathcal{L}^{2}}, \quad \mu_{i}=\frac{3 c k T \zeta_{i} t_{0}}{h^{2} \rho}, \quad i=1,2,3, \quad \theta=\frac{L^{2}}{\mathcal{L}^{2}}$.

$\alpha$ measures the strength of elastic energy relative to kinetic energy; $R e$ is the solvent Reynolds number; $E r$ is the Ericksen number which measures the relative strength of the short-range nematic potential and the isotropic distortional elasticity potential; $\theta$ measures the degree of anisotropy in the distortional elasticity, with values limited to $[-1, \infty) ; 1 / \mu_{i}, i=1,2,3$ are three nematic Reynolds numbers. We drop the tilde ${ }^{\sim}$ on all variables from now on so that all equations and figures in the following correspond to normalized variables, length, and time scales.

The dimensionless forms of the balance of linear momentum, stress constitutive equation, and the continuity equation (dimensional forms are in [33]) take the following form.

Linear momentum balance:

$$
\frac{d}{d t} \mathbf{v}=\nabla \cdot(-p \mathbf{I}+\tau),
$$

where external forces are neglected. 
Continuity equation:

$$
\nabla \cdot \mathbf{v}=0
$$

Constitutive equation for the extra stress:

$$
\begin{aligned}
& \tau=2 \eta \mathbf{D}+a \alpha\left[\mathbf{M}-\frac{\mathbf{I}}{3}-\frac{N}{2}\left(\left(\mathbf{I}+\frac{1}{3 N E r} \Delta\right) \mathbf{M} \cdot \mathbf{M}+\mathbf{M} \cdot\left(\mathbf{I}+\frac{1}{3 N E r} \Delta\right) \mathbf{M}\right.\right. \\
& \left.\left.-2\left(\mathbf{I}+\frac{1}{3 N E r} \Delta\right) \mathbf{M}: \mathbf{M}_{4}\right)\right]-\frac{\alpha}{6 E r}(\Delta \mathbf{M} \cdot \mathbf{M}-\mathbf{M} \cdot \Delta \mathbf{M})-\frac{\alpha}{12 E r}[\nabla \mathbf{M}: \nabla \mathbf{M} \\
& -(\nabla \nabla \mathbf{M}): \mathbf{M}]+\frac{a \alpha \theta}{12 E r}\left[4 \mathbf{M}_{6}:: \nabla \nabla \mathbf{M}+2 \mathbf{M}_{4} \nabla \nabla:: \mathbf{M}_{4}-\nabla \nabla \mathbf{M}: \mathbf{M}_{4}-\left(\nabla \nabla \mathbf{M}: \mathbf{M}_{4}\right)^{T}\right. \\
& \left.-\mathbf{M}_{4} \vdots \nabla \nabla \mathbf{M}-\left(\mathbf{M}_{4} \vdots \nabla \nabla \mathbf{M}\right)^{T}-\left(\mathbf{M} \nabla \nabla \vdots \mathbf{M}_{4}\right)^{T}-\mathbf{M} \nabla \nabla \vdots \mathbf{M}_{4}\right]-\frac{\alpha \theta}{12 E r}\left[\nabla \nabla \mathbf{M} \vdots \mathbf{M}_{4}\right. \\
& \left.-\left(\nabla \nabla \mathbf{M} \vdots \mathbf{M}_{4}\right)^{T}-\mathbf{M}_{4} \vdots \nabla \nabla \mathbf{M}+\left(\mathbf{M}_{4} \vdots \nabla \nabla \mathbf{M}\right)^{T}-\mathbf{M} \nabla \nabla \vdots \mathbf{M}_{4}+\left(\mathbf{M} \nabla \nabla \vdots \mathbf{M}_{4}\right)^{T}\right] \\
& +\left[\mu_{1}(a)(\mathbf{D M}+\mathbf{M D})+\mu_{2}(a) \mathbf{D}: \mathbf{M}_{4}\right],
\end{aligned}
$$

where $\mathbf{M}$ is the second moment of the orientational probability density function of the kinetic theory (called the structure tensor), $\mathbf{M}_{4}$ and $\mathbf{M}_{6}$ are the fourth and sixth moment of the probability density function, respectively, $\eta=1 / R e+\frac{1}{2} \mu_{3}(a)$ and $a=\frac{r^{2}+1}{r^{2}-1}$ parameterizes the aspect ratio $r$ of the spheroidal molecules, where $0<a \leq 1$ corresponds to a rod-like molecule and $-1 \leq a<0$ for platelets [33].

The boundary conditions on velocity $\mathbf{v}$ are scaled to

$$
\left.\mathbf{v}\right|_{y= \pm 1}=( \pm D e, 0,0)
$$

where

$$
D e=\frac{t_{0} v_{0}}{h},
$$

the Deborah number, is the ratio of the relaxation time relative to the time scale set by the moving plates in the shear experiment. Weak shear is defined by a small value of $D e$ indicating the time scale set by the shear experiment is much larger than the molecular relaxation time scale. Following previous studies [8, 11, 12], we assume strong molecular anchoring at the plates given by the quiescent nematic equilibrium of the orientation tensor (the deviatoric part of the structure tensor) $\mathbf{Q}_{0}=\mathbf{M}_{0}-\frac{\mathbf{I}}{3}=$ $s_{0}\left(\mathbf{n n}-\frac{\mathbf{I}}{3}\right)$. The rest state equilibrium of $\mathbf{Q}$ at sufficiently high concentrations is a uniaxial nematic phase, with unique order parameter,

$$
s_{0}=\frac{1}{4}\left[1+3 \sqrt{1-\frac{8}{3 N}}\right] .
$$

The uniaxial director $\mathbf{n}$ is arbitrary for quiescent phases; this degeneracy is broken experimentally by mechanical or chemical plate preparations. We model a uniform plate anchoring condition, either parallel to the flow direction, called tangential anchoring, or perpendicular to the shearing plates, called normal (or homeotropic) anchoring. 
The time evolution equation of $\mathbf{M}$ (in dimensionless form) is given by [33]:

(9)

$$
\left\{\begin{array}{l}
\frac{d}{d t} \mathbf{M}-\Omega \cdot \mathbf{M}+\mathbf{M} \cdot \Omega-a[\mathbf{D} \cdot \mathbf{M}+\mathbf{M} \cdot \mathbf{D}]=-2 a \mathbf{D}: \mathbf{M}_{4} \\
-6\left[\mathbf{Q}-N\left(\mathbf{M} \cdot \mathbf{M}-\mathbf{M}: \mathbf{M}_{4}\right)\right]+\frac{1}{E r}\left[\Delta \mathbf{M} \cdot \mathbf{M}+\mathbf{M} \cdot \Delta \mathbf{M}-2 \Delta \mathbf{M}: \mathbf{M}_{4}\right] \\
+\frac{\theta}{2 E r}\left[(\nabla \nabla \mathbf{M}) \vdots \mathbf{M}_{4}+\left((\nabla \nabla \mathbf{M}) \vdots \mathbf{M}_{4}\right)^{T}+\mathbf{M}_{4} \vdots \nabla \nabla \mathbf{M}+\left(\mathbf{M}_{4} \vdots \nabla \nabla \mathbf{M}\right)^{T}\right. \\
\left.+\mathbf{M} \nabla \nabla \vdots \mathbf{M}_{4}+\left(\mathbf{M} \nabla \nabla \vdots \mathbf{M}_{4}\right)^{T}-4 \mathbf{M}_{6}:: \nabla \nabla \mathbf{M}-2 \mathbf{M}_{4} \nabla \nabla:: \mathbf{M}_{4}\right] .
\end{array}\right.
$$

In order to arrive at a closed system of governing equations at the level of second order tensors, we approximate fourth $\left(\mathbf{M}_{4}\right)$ and sixth $\left(\mathbf{M}_{6}\right)$ order tensors in the above governing system of equations using the following simple closure rules:

$$
\mathrm{M}_{4} \approx \mathrm{MM}, \quad \mathrm{M}_{6} \approx \mathrm{MMM} .
$$

These simple closure approximations respect the traceless property of the orientational dynamic equation, and have been shown to yield a good approximation of kinetic theory in the dynamics of monodomains at the nematic concentrations of interest here $[10,15,16]$. These closures are exact when the molecules are aligned perfectly.

We remark that the distortional elastic free energy reduces to the Oseen-Frank energy after the closure approximation, in which the three Frank elastic constants are given by

$$
k_{1}=k_{2}=\frac{2 k T}{E r} s^{2}\left(1+\frac{\theta}{3}(1-s)\right), \quad k_{3}=\frac{2 k T}{E r} s^{2}\left(1+\frac{\theta}{6}(1+4 s)\right) .
$$

For rod-like NLCPs,

$$
0<k_{1}=k_{2}<k_{3}
$$

whereas for discotic NLCPs (platelets),

$$
0<k_{3}<k_{1}=k_{2} \text {. }
$$

2.1. Asymptotic solutions in weak plane Couette flows. We seek asymptotic solutions of the governing system of equations with the boundary conditions given by (6) and (8). We employ a biaxial representation of the orientation tensor [11]

$$
\mathbf{Q}=s\left(\mathbf{n n}-\frac{1}{3} \mathbf{I}\right)+\beta\left(\mathbf{n}^{\perp} \mathbf{n}^{\perp}-\frac{1}{3} \mathbf{I}\right),
$$

where $(s, \beta)$ are two order parameters measuring the birefringence relative to the optical axes (also called directors) $\mathbf{n}$ and $\mathbf{n}^{\perp}$ confined to the shearing plane $(x, y)$ and parameterized by a director angle $\psi$,

$$
\mathbf{n}=(\cos \psi, \sin \psi, 0), \quad \mathbf{n}^{\perp}=(-\sin \psi, \cos \psi, 0),
$$

and $\mathbf{I}$ is the $3 \times 3$ identity matrix. We propose the solution ansatz

$$
v_{x}=\sum_{k=1}^{\infty} D e^{k} v_{x}^{(k)}, \quad(\bullet)=\sum_{k=0}^{\infty}(\bullet)_{k} D e^{k}, \quad \psi=\psi_{0}+\sum_{k=1}^{\infty} \psi^{(k)} D e^{k},
$$

where $(\bullet)$ represents the order parameters $s, \beta$, respectively. The solution is sensitive to the choice of boundary conditions, so we present tangential $\left(\psi_{0}=0\right)$ and normal $\left(\psi_{0}=\frac{\pi}{2}\right)$ anchoring conditions separately. 
2.2. Tangential anchoring $\left(\psi_{0}=0\right)$. First, we note that

$$
v_{x}^{(2 k)}=\psi^{(2 k)}=s_{2 k-1}=\beta_{2 k-1}=0, \quad k=1, \ldots, \infty,
$$

demanded by the boundary conditions and the governing equations at the respective orders. This also applies to the case of normal anchoring, but not to tilted anchoring $\left(\psi_{0} \neq 0, \frac{\pi}{2}\right)[14]$. The governing equations at order $O(1)$ give the equilibrium solution of $\mathbf{Q}$ consistent with the boundary anchoring condition; the equations at order $O(D e)$ are obtained by solving the following equations for $\psi^{(1)}$ and $v_{x}^{(1)}$ :

$$
\begin{aligned}
& \frac{\partial \psi^{(1)}}{\partial t}=A \frac{\partial^{2} \psi^{(1)}}{\partial y^{2}}+B \frac{\partial v_{x}^{(1)}}{\partial y}, \\
& \frac{\partial v_{x}^{(1)}}{\partial t}=\frac{\partial \tau_{x y}}{\partial y}, \\
& \tau_{x y}=C \frac{\partial^{2} \psi^{(1)}}{\partial y^{2}}+D \frac{\partial v_{x}^{(1)}}{\partial y},
\end{aligned}
$$

where

$$
\begin{aligned}
& A=\frac{1}{9 E r}\left(s_{0}+2\right)\left(3+\theta\left(1-s_{0}\right)\right), \quad B=\frac{1}{2}\left(\lambda_{L}-1\right), \\
& C=-\frac{\alpha s_{0}^{2}}{18 E r}\left[\theta\left(1-s_{0}\right) \lambda_{L}+3\left(\lambda_{L}-1\right)\right], \quad D=\frac{1}{3}\left(\mu_{1} s_{0}+3 \eta\right),
\end{aligned}
$$

where the "tumbling parameter" $\lambda_{L}$ is defined by

$$
\lambda_{L}=\frac{a\left(2+s_{0}\right)}{3 s_{0}} ;
$$

$\left|\lambda_{L}\right|>1$ corresponds to flow aligning and $\left|\lambda_{L}\right|<1$ yields director tumbling in monodomain shear flows $(E r \rightarrow \infty)[14]$.

2.2.1. Steady state features of the major director $\psi^{(1)}$ and primary flow $\boldsymbol{v}_{\boldsymbol{x}}^{(1)}$. The nonzero leading order steady solution for the velocity, order parameters $s$ and $\beta$, and the director angle $\psi$ can be solved explicitly:

$$
\begin{gathered}
v_{x}^{(1)}(y)=y, \quad s_{0}=s_{0}, \quad \beta_{0}=0, \quad \psi^{(1)}(y)=\operatorname{MEr}\left(y^{2}-1\right), \\
M=\frac{9}{4\left(s_{0}+2\right)\left(3+\left(1-s_{0}\right) \theta\right)}\left(1-\lambda_{L}\right) .
\end{gathered}
$$

Note that, as in the isotropic elasticity limit [14], these solvability conditions imply simple shear flow at leading order in $D e$, and yield that the orientational distribution is dominated by nematic (director) distortions. The prefactor (22) yields that the winding number of the major director between the plates is proportional to the Ericksen number, as with the isotropic elasticity limit [14]. The formula (22) yields the scaling law for elastic distortions which are nonuniform across the gap with lengthscale proportional to $M^{-1}$, which in turn is proscribed by three material parameters: $(a, N, \theta)$. For fixed $(a, N),|M|$ decreases as $\theta$ increases $(\theta \in[-1, \infty))$. We summarize the dependence of $M$ on $\theta$ for given material parameters $(a, N)$ as follows:

- The sign of M governs the "chirality" of nematic distortion, or direction of director rotation from the plates. $\mathrm{M}$ is negative for flow-aligning rods $(a>$ $\left.0, \lambda_{L}>1\right)$ and positive for tumbling rods $\left(a>0,0<\lambda_{L}<1\right)$ and discs or platelets $(a<0)$. $|M|$ decreases with respect to all $\theta \in[-1, \infty)$. 

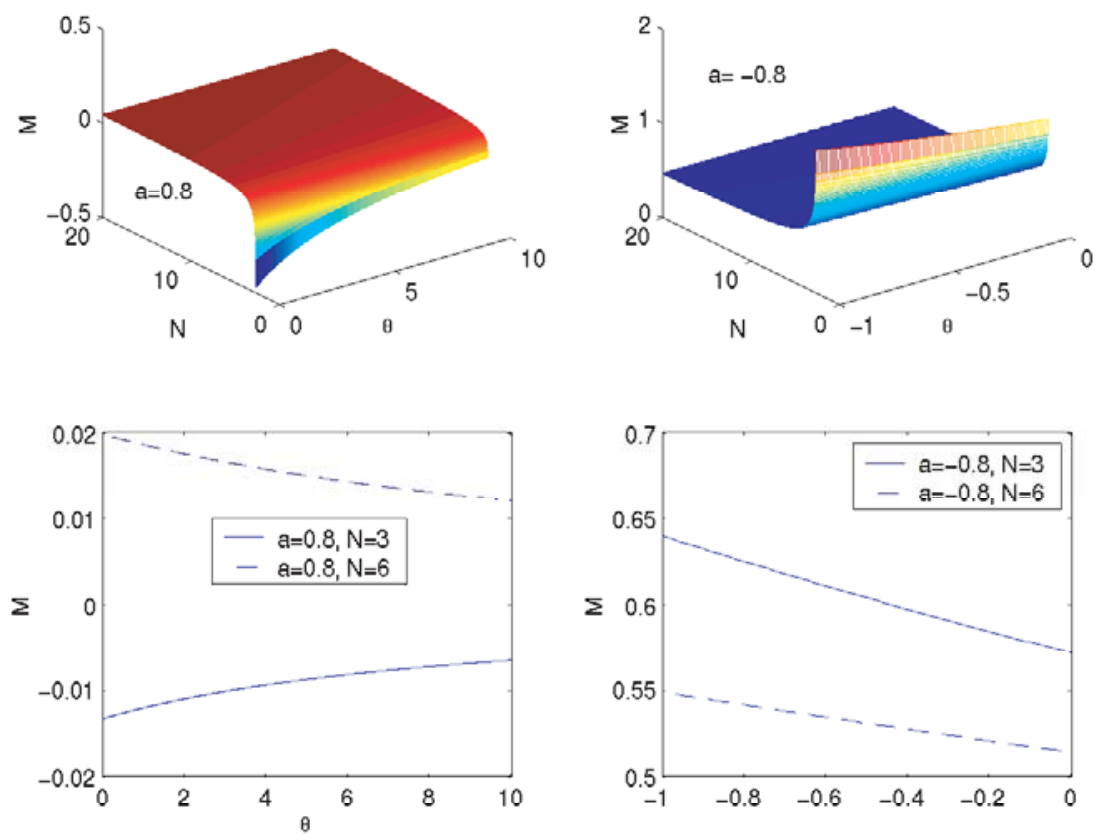

FIG. 2. $M$ as a function of $N$ and $\theta$ with tangentially anchored boundary conditions in weak Couette flow: The top left panel is for rods, with $a=0.8$, whereas the top right panel is for platelets, with $a=-0.8$. The bottom panels show $M$ for two distinct concentrations of rods (left) and platelets (right).

- Physically, the director angle winds counterclockwise for rods in the flowaligning regime $\lambda_{L}>1$ from the lower plate to the midplane, then unwinds from the midplane to the upper plane; the orientation reverses for rods in the tumbling regime and for platelets in all regimes.

- At fixed Ericksen number, anisotropic elasticity tends to reduce the magnitude of the director winding for rods while enhancing director distortion for platelets. Figure 2 depicts $M$ as a function of $(N, \theta)$ two values of $a$ corresponding to rods $(a=0.8)$ and platelets $(a=-0.8)$. Next, we consider a limited notion of stability of this steady-state structure, by studying transients of the first order governing system of equations in the presence of superimposed spatial disturbances.

2.2.2. Transient behavior of $\left(v_{x}^{(1)}, \psi^{(1)}\right)$ near steady states. The transient solution for $v_{x}^{(1)}$ and $\psi^{(1)}$ (the difference between the time-dependent solution and the steady state) obeys the same homogeneous linear partial differential equations but satisfies a zero boundary condition. Its behavior dictates the stability of the steady state within the asymptotic balance model: the steady state is asymptotically stable if the transient solution vanishes as $t \rightarrow \infty$.

Proposition 1. The steady solution $(21,22)$ of system $(18)$ is stable for $A D-$ $B C>0$ and unstable for $A D-B C<0$ with respect to zero boundary conditions on $v_{x}^{(1)}$ and $\phi^{(1)}$. 
Proof. We first consider the case of $B C \neq 0$ and prove the steady solution is stable provided $A D-B C>0$. In the following proof, we drop the superscripts on $\psi$ and $v_{x}$. Extending $(18)_{1}$ to the boundary and accounting for the boundary condition $\psi(-1, t)=\psi(1, t)=0$, we have

$$
\left.\left(A \frac{\partial^{2} \psi}{\partial y^{2}}+B \frac{\partial v_{x}}{\partial y}\right)\right|_{y= \pm 1}=0 .
$$

We introduce a nonnegative functional

$$
I(t)=\int_{-1}^{1}\left[\delta_{1} \psi_{y}^{2}+\delta_{2} v_{x}^{2}\right] d y
$$

with $\delta_{1}>0$ and $\delta_{2}>0$. We note that $A>0$ and $D>0$ from (19).

Case 1. $B C<0$. Choosing $\delta_{1}=|C|$ and $\delta_{2}=|B|$ and integrating by parts, the time derivative of the nonnegative functional can be estimated:

$$
\begin{aligned}
& \frac{d I(t)}{d t}=-2 \int_{-1}^{1}\left[\delta_{1} A \psi_{y y}^{2}+\left(\delta_{1} B+\delta_{2} C\right) \psi_{y y} v_{x, y}+\delta_{2} D v_{x, y}^{2}\right] d y \\
& =-2 \int_{-1}^{1}\left[|C| A \psi_{y y}^{2}+|B| D v_{x, y}^{2}\right] d y<0 .
\end{aligned}
$$

This shows that the steady solution of the system is stable.

Case 2. Choose $\delta_{1}=\max \left(\frac{C^{2}}{A D}, 1\right) \geq 1, \delta_{2}=\max \left(\frac{B^{2}}{A D}, 1\right) \geq 1$. We have

$$
\begin{aligned}
& \frac{d I(t)}{d t}=2 \int_{-1}^{1}\left[\delta_{1} \psi_{y} \psi_{t y}+\delta_{2} v_{x} v_{x t}\right] d y \\
& =-2 \int_{-1}^{1}\left[\delta_{1} A \psi_{y y}^{2}+\left(\delta_{1} B+\delta_{2} C\right) \psi_{y y} v_{x, y}+\delta_{2} D v_{x, y}^{2}\right] d y .
\end{aligned}
$$

The integrand is quadratic and the discriminant is

$$
\begin{aligned}
& \left(\delta_{1} B+\delta_{2} C\right)^{2}-4 \delta_{1} A \delta_{2} D \\
= & \delta_{1} A D\left(\frac{B^{2}}{A D} \delta_{1}-\delta_{2}\right)+\delta_{2} A D\left(\frac{C^{2}}{A D} \delta_{2}-\delta_{1}\right)-2 \delta_{1} \delta_{2}(A D-B C) \\
= & \delta_{1} A D\left[\frac{B^{2}}{A D} \max \left(\frac{C^{2}}{A D}, 1\right)-\max \left(\frac{B^{2}}{A D}, 1\right)\right] \\
& +\delta_{2} A D\left[\frac{C^{2}}{A D} \max \left(\frac{B^{2}}{A D}, 1\right)-\max \left(\frac{C^{2}}{A D}, 1\right)\right] \\
& -2 \delta_{1} \delta_{2}(A D-B C)<-2 \delta_{1} \delta_{2}(A D-B C)<0 .
\end{aligned}
$$

The first inequality is based on $\frac{B^{2} C^{2}}{(A D)^{2}}<1$ because of $A D-B C>0$ and $B C>0$. Since $\delta_{1} A>0$, the integrand is always positive; thus $\frac{d I(t)}{d t}<0$. Hence the steady solution of the system is stable. 
The proof for $B C=0$ is far simpler and omitted. To prove instability when $A D-B C<0$, we only need to find one unstable mode. Let $\phi(y, t)=\psi_{y}(y, t)$; the system (18) with the boundary condition becomes

$$
\begin{aligned}
& \phi_{t}=A \phi_{y y}+B v_{x, y y}, \\
& v_{x, t}=C \phi_{y y}+D v_{x, y y}, \\
& \left.\left(A \phi_{y}+B v_{x, y}\right)\right|_{y= \pm 1}=0, \quad v_{x}(1, t)=1, \quad v_{x}(-1, t)=-1 .
\end{aligned}
$$

To find an unstable mode, we seek normal modes of the form

$$
\left(\begin{array}{c}
\phi \\
v_{x}
\end{array}\right)=e^{\gamma t}\left(\begin{array}{c}
\tilde{\phi}(y) \\
\tilde{v}_{x}(y)
\end{array}\right)
$$

and consider the resultant eigenvalue problem

$$
P\left(\begin{array}{c}
\tilde{\phi} \\
\tilde{v_{x}}
\end{array}\right)_{y y}=\gamma\left(\begin{array}{c}
\tilde{\phi} \\
\tilde{v_{x}}
\end{array}\right), \quad P=\left(\begin{array}{cc}
A & B \\
C & D
\end{array}\right),
$$

where $\gamma$ is the growth rate. The steady solution is unstable if $\gamma>0$.

The matrix $P$ has two distinct eigenvalues given by

$$
r_{1}=\frac{A+D-\sqrt{(A+D)^{2}-4(A D-B C)}}{2}<0, \quad r_{2}=\frac{A+D+\sqrt{(A+D)^{2}-4(A D-B C)}}{2}>0 .
$$

Let $\xi=\arctan \frac{A-r_{1}}{B}$, then

$$
\left(\begin{array}{c}
\tilde{\phi} \\
\tilde{v_{x}}
\end{array}\right)=\left(\begin{array}{cc}
\cos \xi & \sin \xi \\
-\sin \xi & \cos \xi
\end{array}\right)\left(\begin{array}{c}
\cos \xi \frac{\sin \sqrt{\frac{\gamma}{-r_{1}}} y}{\sin \sqrt{\frac{\gamma}{-r_{1}}}} \\
\sin \xi \frac{\sinh \sqrt{\frac{\gamma}{r_{2}}} y}{\sinh \sqrt{\frac{\gamma}{r_{2}}}}
\end{array}\right)
$$

is a solution of $(28)$ satisfying $v_{x}(1, t)=v_{x}(-1, t)=0$ and $\gamma$ is determined by $\left(A \phi_{y}+\right.$ $\left.B v_{x, y}\right)\left.\right|_{y= \pm 1}=0$, which yields

$$
\left(A-r_{1}\right)\left(1+\frac{A\left(A-r_{1}\right)}{B^{2}}\right) \operatorname{coth} \sqrt{\frac{\gamma}{r_{2}}}-\sqrt{-r_{1} r_{2}} \cot \sqrt{\frac{\gamma}{-r_{1}}}=0 .
$$

As $\gamma \rightarrow+\infty$, the first term goes to a finite value while the second one is periodic and varies between $-\infty$ and $+\infty$ within one period. Consequently, the equation has infinitely many positive solutions for $\gamma$, which completes the proof, and indicates that the diagnostic $A D-B C$ signals catastrophic instability when it is negative.

We note that for discs $(a<0)$ and flow-aligning rods $\left(a>0, \lambda_{L}>1\right)$,

$$
\begin{aligned}
& A D-B C=\frac{1}{27 E r}\left[3\left(2+s_{0}\right)\left(\mu_{1} s_{0}+3 \eta\right)+\frac{\alpha}{4}\left(\left(\lambda_{L}-1\right) 3 s_{0}\right)^{2}\right. \\
& \left.+\theta\left(1-s_{0}\right)\left(2+s_{0}\right)\left(\frac{\alpha}{12} a\left(\left(\lambda_{L}-1\right) 3 s_{0}\right)+\mu_{1} s_{0}+3 \eta\right)\right]>0 .
\end{aligned}
$$

Hence, the steady state is always stable for discotic LCPs and flow-aligning rods, and may be unstable only for tumbling rods $\left(0<a, 0<\lambda_{L}<1\right)$. 


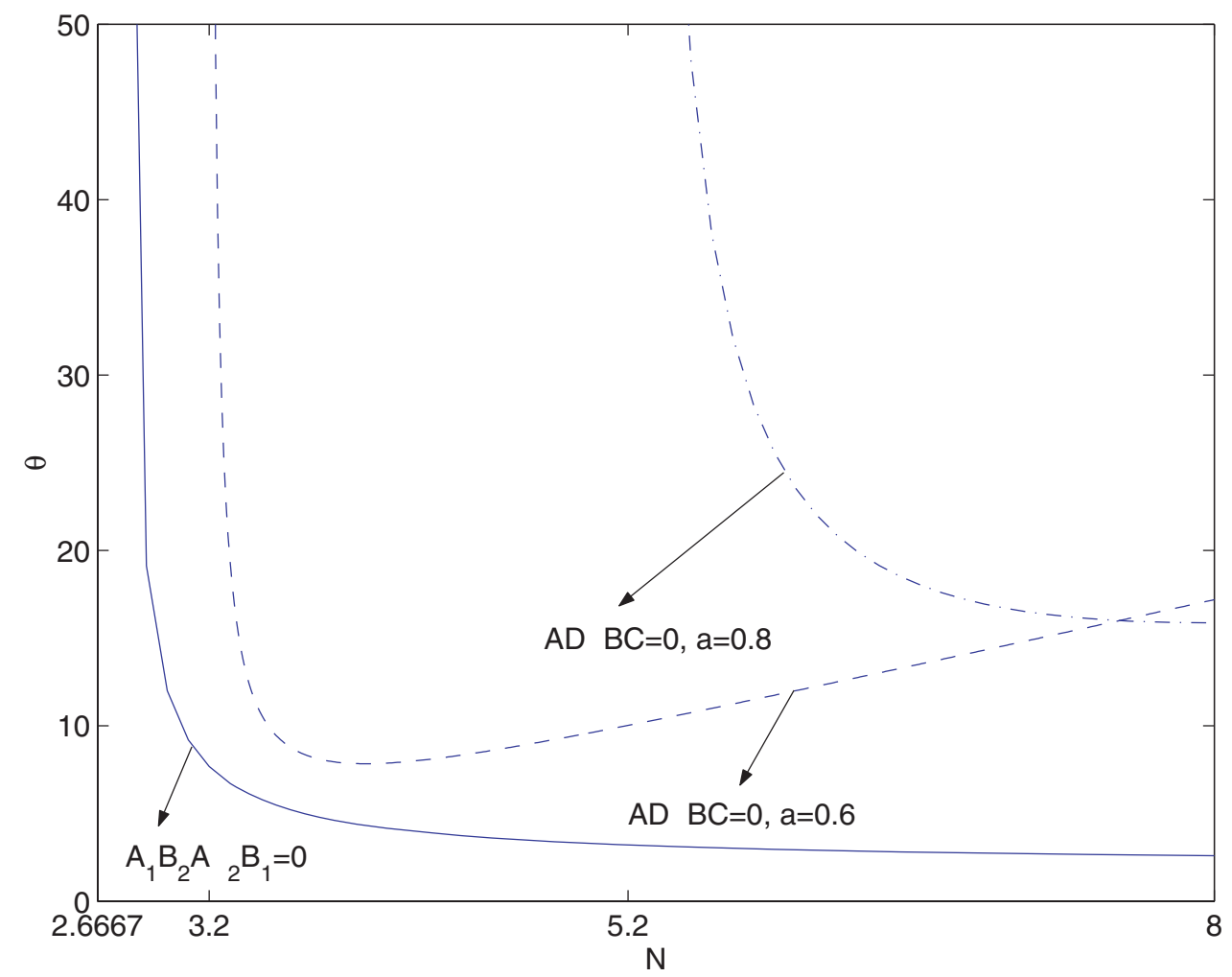

Fig. 3. The neutral stability curves defined by $A_{1} B_{2}-A_{2} B_{1}=0$ and $A D-B C=0$ with tangentially anchored boundary conditions in weak shear. The arrows point to the positive directions of the discriminant. The systems for $\left(s_{2}, \beta_{2}\right)$ and $\left(\psi^{(1)}, v_{x}^{(1)}\right)$ are stable when the discriminant is positive, respectively, unstable otherwise. The parameter values are $\mathrm{Er}=100, \alpha=1, \mu_{1}=0.001$ and $\eta=0.002$.

The neutral stability curve $(A D-B C=0)$ depends on the values of the parameters $\left(\theta, a, N, \alpha, \mu_{1}, \eta\right)$. From (34), $A D-B C>0$ can be translated into two separate constraints on the energy parameter $\alpha$ and $\theta$,

$$
\begin{aligned}
& \alpha \leq \alpha_{c}=\frac{4\left(\mu_{1} s_{0}+3 \eta\right)}{a\left(1-\lambda_{L}\right) s_{0}} ; \text { or } \\
& \alpha>\alpha_{c} \text { and } \theta<\theta_{c}=\frac{3\left(2+s_{0}\right)\left(\mu_{1} s_{0}+3 \eta\right)+\frac{\alpha}{4}\left(\left(\lambda_{L}-1\right) 3 s_{0}\right)^{2}}{\left(1-s_{0}\right)\left(2+s_{0}\right)\left(\frac{\alpha}{12} a\left(\left(1-\lambda_{L}\right) 3 s_{0}\right)-\mu_{1} s_{0}+3 \eta\right)} .
\end{aligned}
$$

Figure 3 depicts the stability transition curve in the parameter space $(N, \theta)$ at a few selected values of other parameters. We observe that the values of $N, \theta$ that yield instability tend to be large and out of the practical range for nematic polymer materials. We also note that the flow-aligning region and the stable region versus $(N, \theta)$ both grow significantly as the shape parameter $a$ increases, i.e., as the aspect ratio becomes more extreme. The instability region vanishes as $a \rightarrow \frac{3 s_{0}}{2+s_{0}}$. The stable region also grows as $\mu_{1} / \alpha, \eta / \alpha$ increase.

We summarize this more precise statement of Proposition 1 in the following corollary.

COROLlary 1 . The steady asymptotic solution $(21,22)$ is asymptotically stable within the leading order balance equations if either of conditions (35) is satisfied. If 
$\alpha>\alpha_{c}$ and $\theta>\theta_{c}$, where $\alpha_{c}, \theta_{c}$ are defined in (35), the steady solution is unstable and the leading order system of equations is ill-posed.

2.2.3. Steady state features of the order parameters $s_{2}$ and $\beta_{2}$ at $O\left(D e^{\mathbf{2}}\right)$. The order parameters $(s, \beta)$ vanish at leading order in $D e$, with order $O\left(D e^{2}\right)$ behavior governed by the equations

$$
\begin{aligned}
& \frac{\partial s_{2}}{\partial t}=\frac{-1}{9 E r}\left(A_{1} \frac{\partial^{2} s_{2}}{\partial y^{2}}+B_{1} \frac{\partial^{2} \beta_{2}}{\partial y^{2}}+C_{1} s_{2}+D_{1} \beta_{2}+E_{1}\left(\frac{\partial \psi^{(1)}}{\partial y}\right)^{2}+\right. \\
& \left.F_{1} \psi_{1} \frac{\partial^{2} \psi^{(1)}}{\partial y^{2}}+G_{1} \psi^{(1)} \frac{\partial v_{x}^{(1)}}{\partial y}\right)+2 s_{0} \psi^{(1)} \frac{\partial \psi^{(1)}}{\partial t}, \\
& \frac{\partial \beta_{2}}{\partial t}=\frac{-1}{9 E r}\left(A_{2} \frac{\partial^{2} s_{2}}{\partial y^{2}}+B_{2} \frac{\partial^{2} \beta_{2}}{\partial y^{2}}+C_{2} s_{2}+D_{2} \beta_{2}+E_{2}\left(\frac{\partial \psi^{(1)}}{\partial y}\right)^{2}+\right. \\
& \left.F_{2} \psi^{(1)} \frac{\partial^{2} \psi^{(1)}}{\partial y^{2}}+G_{2} \psi^{(1)} \frac{\partial v_{x}^{(1)}}{\partial y}\right)-2 s_{0} \psi^{(1)} \frac{\partial \psi^{(1)}}{\partial t}
\end{aligned}
$$

where the coefficients are lengthy and provided in Appendix A. This system of equations is linear in $\left(s_{2}, \beta_{2}\right)$ but driven by nonlinear functions of $v_{x}^{(1)}$ and $\psi^{(1)}$.

The steady solution, with $\Lambda$ and $\Gamma$ defined in the appendix, is

$$
\begin{aligned}
& \beta_{2}(y)=K_{1}\left(\frac{\cosh (\sqrt{E r} \Lambda y)}{\cosh (\sqrt{E r} \Lambda)}-1\right)+K_{2}\left(\frac{\cosh (\sqrt{E r} \Gamma y)}{\cosh (\sqrt{E r} \Gamma)}-1\right)+R_{1} \operatorname{Er}\left(y^{2}-1\right), \\
& s_{2}(y)=K_{3}\left(\frac{\cosh (\sqrt{E r} \Lambda y)}{\cosh (\sqrt{E r} \Lambda)}-1\right)+K_{4}\left(\frac{\cosh (\sqrt{E r} \Gamma y)}{\cosh (\sqrt{E r} \Gamma)}-1\right)+S_{1} \operatorname{Er}\left(y^{2}-1\right),
\end{aligned}
$$

where the coefficients are given in Appendix B.

We denote $\lambda^{2}=1 /(\operatorname{Er} \Lambda)$ and $\mu^{2}=1 /(\operatorname{Er} \Gamma) ; \lambda^{2}$ is always positive and concave up as a function of $\theta$, whereas $\mu^{2}$ is monotonically decreasing and may change sign as $\theta$ varies. For example, $\mu^{2}$ goes through zero at a critical degree of anisotropy $\theta_{c}=2.93$ for $N=6$. For $\theta>\theta_{c}$, the steady state becomes highly oscillatory; we show in the study of transient solutions that this behavior coincides with the onset of ill-posedness in the governing system of equations. The behavior of $\theta_{c}$ versus concentration $N$ can be gleaned from Figure 3.

Since the dominating terms in the order parameters near $y= \pm 1$ are

$$
\frac{e^{\sqrt{E r} \Lambda y}}{e^{\sqrt{E r} \Lambda}}, \quad \frac{e^{\sqrt{E r} \Gamma y}}{e^{\sqrt{E r} \Gamma}},
$$

the order parameters have a boundary layer near the wall, whose width is proportional to

$$
\frac{1}{\sqrt{E r} \Lambda}, \quad \frac{1}{\sqrt{E r} \Gamma},
$$

respectively. These are the penetration depths of the wall layer for tangential anchoring, which agrees with the asymptotic analysis of the DMG model [14] in the single elastic constant limit. One finds the order parameters are coupled with anisotropic elasticity, i.e., the orientational distribution is strongly biaxial (birefringent in any plane). For both rods and discs, by comparing the two exponential terms in $\beta_{2}$ and $s_{2}$, we notice that the boundary layer in $s_{2}$ is governed by $\frac{1}{\sqrt{E r} \Lambda}$, whereas in $\beta_{2}$ by $\frac{1}{\sqrt{E r} \Gamma}$, so that their scaling behavior is incommensurate with the leading order wall layer scaling. 
TABLE 1

Steady state features of the order parameter morphology for Couette flow with tangential anchoring (BL denotes boundary layer).

\begin{tabular}{|c|c|c|c|c|}
\hline & FA/rods & FA/discs & T/rods & T/discs \\
\hline$s-s_{0}$ & Concave down & Concave up & Concave up & $\begin{array}{c}\text { Concave down } \\
\text { Concave up in BL }\end{array}$ \\
\hline$\beta$ & Concave up & $\begin{array}{c}\text { Concave up } \\
\text { C Concave down in BL }\end{array}$ & Concave down & Concave down \\
\hline
\end{tabular}

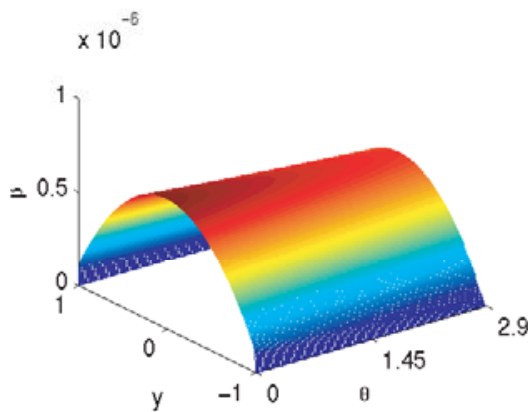

(a)

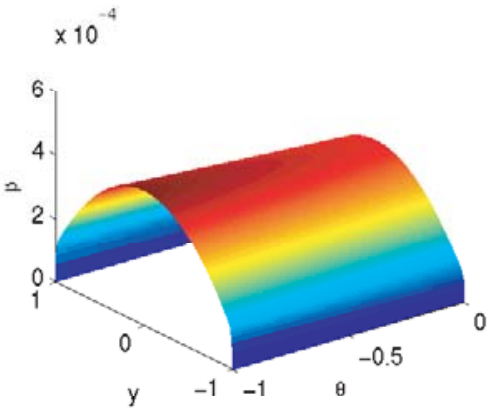

(c)

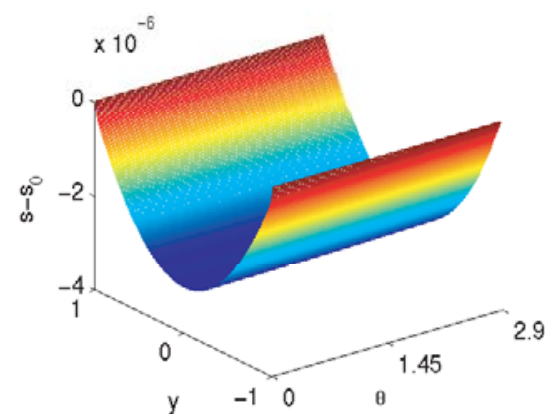

(b)

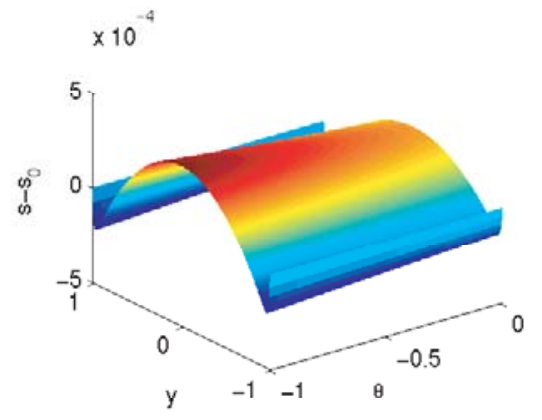

(d)

FIG. 4. The steady-state asymptotic solutions $\beta$ (left column) and $s-s_{0}$ (right column) in the tumbling regime as functions of $(\theta, y)$ with tangentially anchored boundary conditions in weak Couette flows. (a) and (b) depict the solution for rods with parameter values $a=0.8, N=6$, De $=$ $0.01, E r=100$. (c) and (d) depict the solution for discs with parameter values $a=-0.8, N=$ 6, $D e=0.01, E r=100$.

Table 1 tabulates the general behavior of the steady states in the regime of flowaligning and tumbling for both rods and discs, in which FA stands for flow-aligning, $\mathrm{T}$ denotes tumbling, and BL denotes a boundary layer. The concavity switching phenomenon between tumbling and flow-aligning materials, noted in [14], is observed in both rods and discs. The steady states are in general insensitive to changes in $\theta$. In addition, the order parameter correction $s_{2}$ goes through a similar transition for rods versus discs, whereas $\beta$ has the same concavity; this is another indication of strong biaxiality for anisotropic elasticity. Figure 4 depicts a typical steady state asymptotic solution of $\left(s_{2}, \beta_{2}\right)$ for tumbling rods $(a=0.8)$ and tumbling discs $(a=-0.8)$. 
2.2.4. Transient behavior of $\left(s_{2}, \beta_{2}\right)$ near steady states. The transient behavior of the order parameters $s_{2}$ and $\beta_{2}$ obey:

$$
\begin{aligned}
& \frac{\partial \tilde{s_{2}}}{\partial t}=\frac{-1}{9 E r}\left(A_{1} \frac{\partial^{2} \tilde{s_{2}}}{\partial y^{2}}+B_{1} \frac{\partial^{2} \tilde{\beta_{2}}}{\partial y^{2}}+C_{1} \tilde{s_{2}}+D_{1} \tilde{\beta_{2}}\right), \\
& \frac{\partial \tilde{\beta_{2}}}{\partial t}=\frac{-1}{9 E r}\left(A_{2} \frac{\partial^{2} \tilde{s_{2}}}{\partial y^{2}}+B_{2} \frac{\partial^{2} \tilde{\beta_{2}}}{\partial y^{2}}+C_{2} \tilde{s_{2}}+D_{2} \tilde{\beta_{2}}\right),
\end{aligned}
$$

with zero boundary conditions.

After tedious but straightforward Fourier analysis, the growth $\left(\sigma^{\prime}>0\right)$ or decay $\left(\sigma^{\prime}<0\right)$ of solutions $\tilde{s_{2}}(y, t)$ and $\tilde{\beta_{2}}(y, t)$, due to the time-dependent factor $e^{\sigma^{\prime} t}$, is determined by $\operatorname{sgn}\left(\sigma^{\prime}\right)$

$\sigma_{ \pm}^{\prime}=-\frac{1}{2}\left[C_{1}+D_{2}-A_{1} k^{2}-B_{2} k^{2} \pm\right.$

$\sqrt{\left(C_{1}+D_{2}-A_{1} k^{2}-B_{2} k^{2}\right)^{2}-4\left(\left(A_{2} k^{2}-C_{2}\right)\left(D_{1}-B_{1} k^{2}\right)+\left(C_{1}-A_{1} k^{2}\right)\left(D_{2}-B_{2} k^{2}\right)\right)}$. (41)

We now analyze $\operatorname{sgn}\left(\sigma_{ \pm}^{\prime}\right)$ to deduce stability. For long waves $(|k|<<1)$, asymptotic formulae can be derived:

$$
\sigma_{ \pm}^{\prime} \sim-\frac{1}{2}\left[C_{1}+D_{2} \pm \sqrt{\left(C_{1}+D_{2}\right)^{2}+4\left(C_{2} D_{1}-C_{1} D_{2}\right)}\right] .
$$

Using the formulae in Appendix $\mathrm{B}, C_{1}, D_{2}>0, C_{2}=0$, which implies $\sigma_{ \pm}^{\prime}<0$. On the other hand, the growth rate for short waves $(|k|>>1)$ is dominated by

$$
\sigma_{ \pm}^{\prime} \sim \frac{1}{2}\left[A_{1}+B_{2} \pm \sqrt{\left(A_{1}+B_{2}\right)^{2}+4\left(A_{2} B_{1}-A_{1} B_{2}\right)}\right] k^{2} .
$$

We find $\sigma_{ \pm}^{\prime}<0$ only for sufficiently small $|\theta|$. Otherwise, $A_{1} B_{2}-A_{2} B_{1}$ may be negative, leading to a positive growth rate proportional to $k^{2}$, an ill-posed behavior. The transition to ill-posedness, $A_{1} B_{2}-A_{2} B_{1}=0$, simplifies dramatically to the condition $\theta=\frac{6}{5 s_{0}-2}$, where $s_{0}$ is given in (8). This neutral stability curve is plotted in Figure 3.

Proposition 2. The steady state solution $\left(s_{2}, \beta_{2}\right)$ of $(36)$ is catastrophically unstable if and only if the degree of anisotropic elasticity satisfies

$$
\theta>\frac{6}{5 s_{0}-2} .
$$

2.2.5. Rheological features of steady structures. The shear viscosity (shear stress divided by local shear rate) at the plates is identical to the averaged shear viscosity over the shear cell; it is a nonzero constant at $O(D e)$, given by

$$
\eta_{\text {wall }}=\frac{\tau_{x y}}{\frac{d v_{x}^{(1)}}{d y}}=\frac{\alpha a^{2}\left(1-\frac{1}{\lambda_{L}}\right)\left[\theta\left(1-s_{0}\right)+3\left(1-\frac{1}{\lambda_{L}}\right)\right]}{36\left(3+\theta\left(1-s_{0}\right)\right)}+\frac{1}{3}\left(\mu_{1} s_{0}+3 \eta\right) .
$$

It can be readily shown that $\eta_{\text {wall }}$ is a slowly varying, decreasing function of the degree of anisotropic elasticity $\theta$ for tumbling rods and all platelet nematic liquids; however, $\eta_{\text {wall }}$ increases versus $\theta$ for flow-aligning rods. Nonzero first normal stress difference $N_{1}$ and second normal stress difference $N_{2}$ show up at order $O\left(D e^{2}\right)$ and 
are given in Appendix C. These non-Newtonian effects are measurable physically, e.g., $N_{1}>0$ corresponds to pushing the parallel plates apart and $N_{1}<0$ corresponds to pulling the plates together. $N_{1}$ is a linear combination of $\alpha$ and $\mu_{2}$ and $N_{2}$ is a linear combination of $\alpha$ and $\mu_{1}+\mu_{2}$. At walls, the terms containing $\mu_{1,2}$ drop out so that $N_{1}$ and $N_{2}$ are proportional to $\alpha$ :

$$
\begin{aligned}
N_{1}= & {\left[\frac{G}{54 E r}\left(K_{3} \lambda^{2}+K_{4} \mu^{2}+2 S\right)\right.} \\
& +\frac{\left(-4 a \theta+3 s_{0}+16 a \theta s_{0}^{3}-12 a+12 a s_{0}^{2}-12 a \theta s_{0}\right)}{108 E r}\left(K_{1} \lambda^{2}+K_{2} \mu^{2}+2 R\right) \\
& \left.+\frac{4 K^{2} s_{0}}{27 E r}\left(-4 a \theta s_{0}^{2}-4 a \theta+24 a \theta s_{0}^{3}-6 a s_{0}+9 s_{0}+18 a s_{0}^{2}-12 a-16 a \theta s_{0}\right)\right] \alpha, \\
N_{2}= & -\frac{G}{54 E r}\left(K_{3} \lambda^{2}+K_{4} \mu^{2}+2 S\right) \\
& +\frac{\left(8 a \theta+12 a-3+8 a s_{0}-16 a s_{0}^{2}-12 s_{0}\right) s_{0}}{108 E r}\left(K_{1} \lambda^{2}+K_{2} \mu^{2}+2 R\right) \\
& \left.+\frac{4 K^{2} a s_{0}}{27 E r}\left(8 \theta s_{0}^{2}+2 \theta-24 \theta s_{0}^{3}+12 s_{0}-9 s_{0}-18 a s_{0}^{2}+6+14 \theta s_{0}\right)\right] \alpha,
\end{aligned}
$$

where $G, K, R, S, \lambda, \mu$ and $K_{i}(i=1,2,3,4)$ are given in Appendix C.

Figure 5 depicts $N_{1}$ and $N_{2}$ for tumbling rods as well as discs as functions of $y$ at some parameter values; Figure 6 shows $N_{1}$ and $N_{2}$ for flow-aligning rods and discs. Table 3 lists the averaged normal stress differences calculated in four representative cases. We summarize the noticeable features in the stress differences below.

- For flow-aligning rods, $N_{1}<0$ and $N_{2}>0$ across the gap; their signs change in plate boundary layers for small degree $\theta$ of elastic anisotropy.

- These properties reverse for flow-aligning or tumbling discs, which may experience sign changes in $N_{1}$ and $N_{2}$ in the middle of the plate gap for small $|\theta|$.

- For tumbling rods and all discotic NLCPs, $N_{1}>0$ and $N_{2}<0$, with sign changes for rods in the wall boundary layer for large $\theta$, and sign changes for platelets in the midgap at small $|\theta|$.

- The averages across the gap yield $N_{1}>0$ and $N_{2}<0$ for flow-aligning rods, and $N_{1}<0$ and $N_{2}>0$ in all other cases.

2.3. Homeotropic anchoring $\left(\psi_{0}=\frac{\pi}{2}\right)$. The boundary anchoring condition affects only the coefficients of the governing system of partial differential equations at each order. The structure of the equations at $O(D e)$ for $\left(v_{x}^{(1)}, \psi^{(1)}\right)$ are identical to (18) with the following new coefficients:

$$
\begin{aligned}
& A=\frac{1}{9 E r}\left(s_{0}+2\right)\left(3+\theta\left(2 s_{0}+1\right)\right), \quad B=-\frac{1}{2}\left(1+\lambda_{L}\right), \\
& C=\frac{\alpha s_{0}}{18 E r}\left[\theta\left(2 s_{0}+1\right) \lambda_{L}+3\left(\lambda_{L}+1\right)\right], \quad D=\frac{1}{3}\left(\mu_{1} s_{0}+3 \eta\right) .
\end{aligned}
$$

The nonzero leading order solution for the order parameters, primary velocity component, and major director angle are

$$
\begin{gathered}
s_{0}=s_{0}, \quad \beta_{0}=0, \quad v_{x}^{(1)}(y)=y, \quad \psi^{(1)}(y)=\operatorname{MEr}\left(y^{2}-1\right), \\
M=\frac{9}{2\left(s_{0}+2\right)\left(3+\theta\left(2 s_{0}+1\right)\right)}\left(1+\lambda_{L}\right) .
\end{gathered}
$$




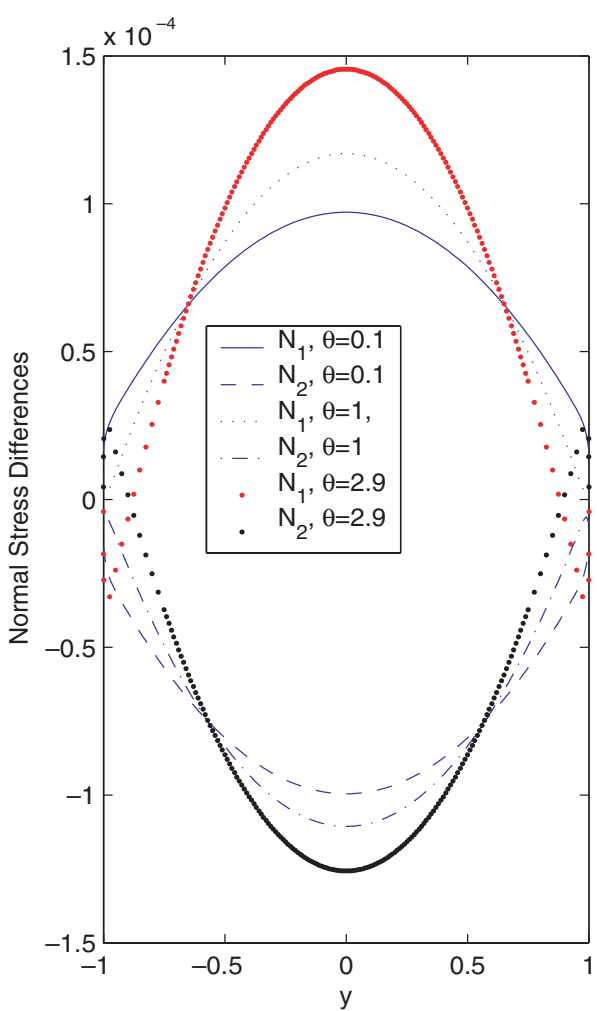

(a)

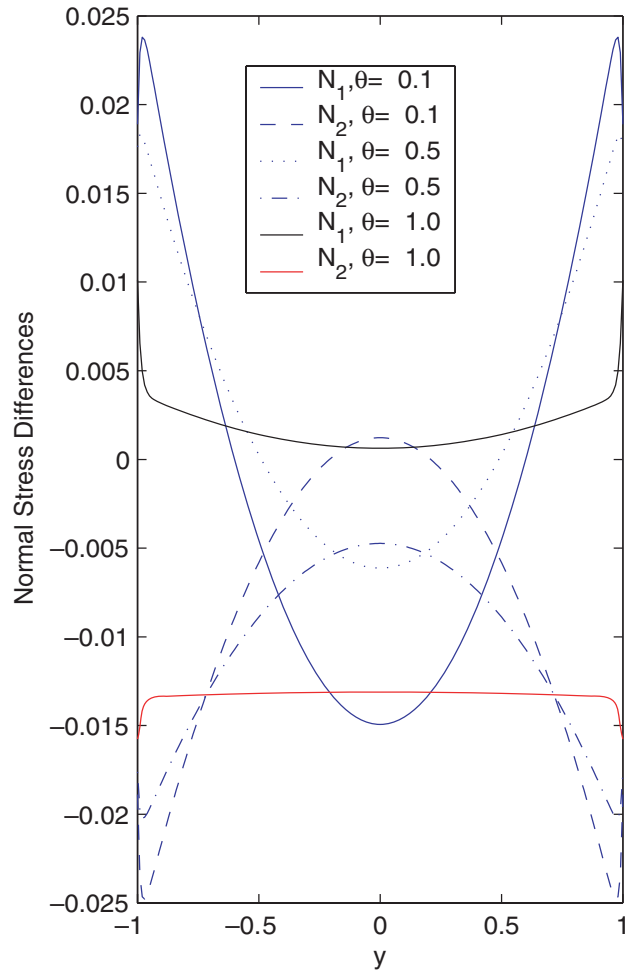

(b)

FIG. 5. The normal stress differences $N_{1}$ and $N_{2}$ in tumbling regimes as functions of $y$ at selected values of $\theta$ with tangentially anchored boundary conditions in weak shear. (a) Rods: $a=$ $0.8, N=6, D e=0.01, E r=100, \alpha=10, \mu_{1}=0.01, \eta=0.02$. (b) Discs: $a=-0.8, N=6, D e=$ $0.01, E r=100, \alpha=10, \mu_{1}=-0.01, \eta=0.02$.

It is easy to see that $|M|$ decreases with respect to $\theta ; M$ is negative for flow-aligning discs $\left(a<0, \lambda_{L}<-1\right)$ and positive for all other cases. Thus, directors wind counterclockwise from the lower shearing plate to the midplane, and unwind from the midplane to the upper plate for flow-aligning discs. The winding reverses direction in the other cases.

Analogous to tangential anchoring, steady states may be catastrophically unstable for discs in the flow-aligning regime $a<0, \lambda_{L}<-1$ if $A D-B C<0$, yet are stable in the other cases, where

$$
\begin{aligned}
A D-B C=\frac{1}{27 E r} & {\left[(s+2)\left(\mu_{1} s_{0}+3 \eta\right)\left(\theta\left(2 s_{0}+1\right)+3\right)\right.} \\
+ & \left.\frac{\alpha s_{0}}{4}\left(1+\lambda_{L}\right)\left(a \theta\left(2 s_{0}+1\right)\left(s_{0}+2\right)+9 s_{0}\left(1+\lambda_{L}\right)\right)\right] .
\end{aligned}
$$

Proposition 3. The steady-state solution is stable so long as

$$
\theta>\theta_{c}=-\frac{12\left(s_{0}+2\right)\left(\mu_{1} s_{0}+3 \eta\right)+9 \alpha s_{0}^{2}\left(1+\lambda_{L}\right)^{2}}{4\left(s_{0}+2\right)\left(2 s_{0}+1\right)\left(\mu_{1} s_{0}+3 \eta\right)+a \alpha s_{0}\left(1+\lambda_{L}\right)\left(1+2 s_{0}\right)\left(2+s_{0}\right)}
$$

The governing system of equations for the order parameters $(s, \beta)$ at order $O\left(D e^{2}\right)$ is of the same form as tangential anchoring, with different coefficients given in Appendix $C$. 


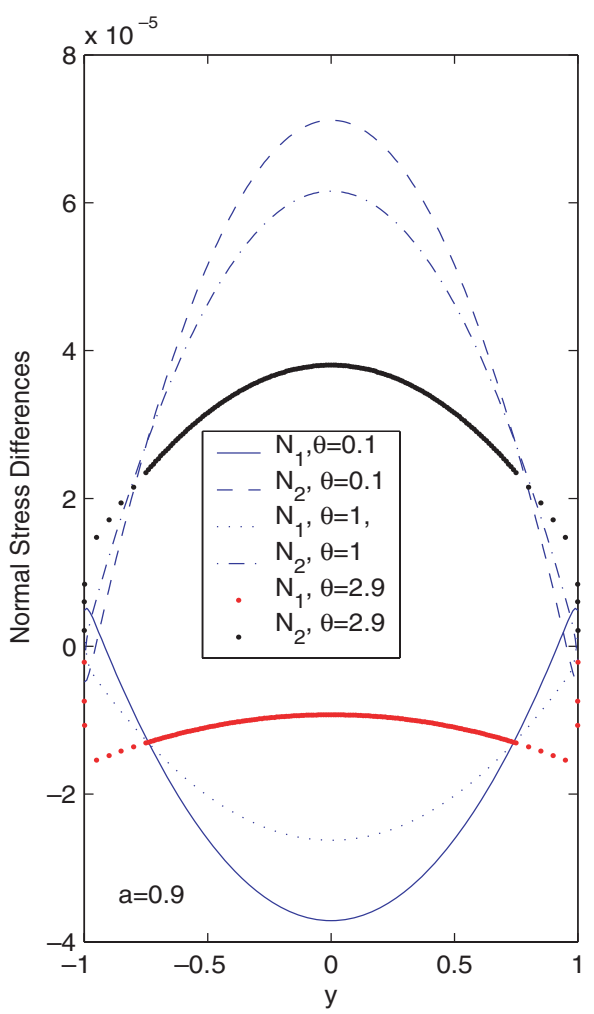

(a)

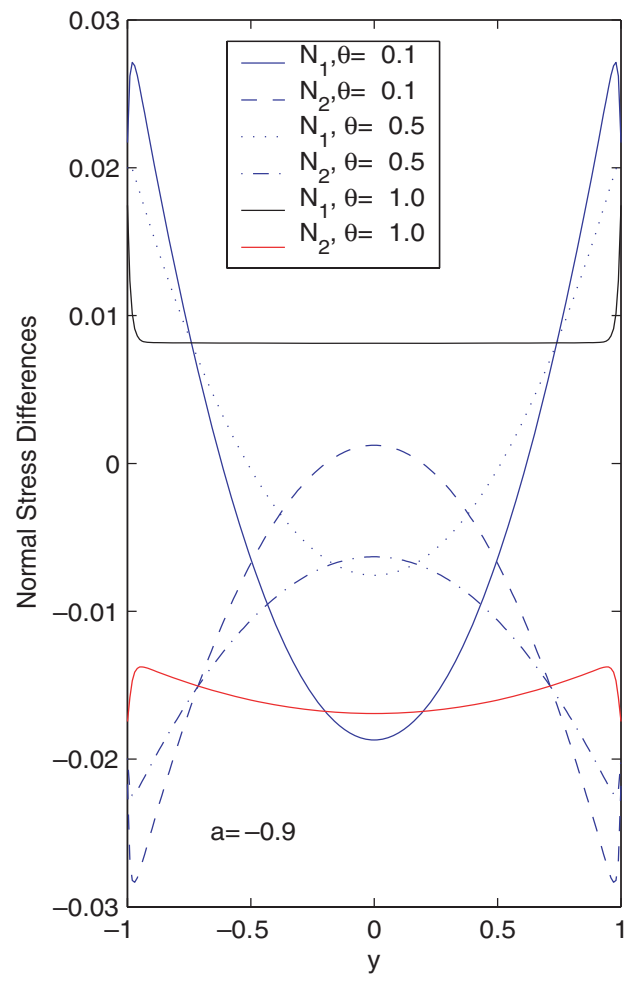

(b)

FIG. 6. The normal stress differences $N_{1}$ and $N_{2}$ in flowing-aligning regimes as functions of $y$ at selected values of $\theta$ with tangentially anchored boundary conditions in weak shear. (a) Rods: $a=0.9, N=6, D e=0.01, E r=100, \alpha=10, \mu_{1}=0.01, \eta=0.02$. (b) Discs: $a=-0.9, N=6, D e=$ $0.01, E r=100, \alpha=10, \mu_{1}=-0.01, \eta=0.02$.

The steady solutions are

$$
\begin{aligned}
& \beta_{2}(y)=K_{11}\left(\frac{\cosh (\sqrt{E r} \Lambda y)}{\cosh (\sqrt{E r} \Lambda)}-1\right)+R_{2} \operatorname{Er}\left(y^{2}-1\right), \\
& s_{2}(y)=K_{21}\left(\frac{\cosh (\sqrt{E r} \Lambda y)}{\cosh (\sqrt{E r} \Lambda)}-1\right)+K_{22}\left(\frac{\cosh (\sqrt{\operatorname{Er}} \Gamma y)}{\cosh (\sqrt{\operatorname{Er}} \Gamma)}-1\right)+S_{2} \operatorname{Er}\left(y^{2}-1\right) .
\end{aligned}
$$

In this solution, $\mu^{2}$ (defined earlier) changes sign only as $\theta$ varies below a threshold value $\theta_{d}$ for platelets. Again, the change of sign in $\mu^{2}$ coincides with ill-posedness in the governing system of equations.

Notice that in $s_{2}$, there are two cosh terms, whereas there is only one in $\beta_{2}$. For rods, the second term in $s_{2}$ dominates in the boundary layer while the first term dominates for discs. Table 2 tabulates features of the steady states. Compared with results above for tangential anchoring, the steady states with normal anchoring are more sensitive to the degree $\theta$ of elastic anisotropy. The order parameter variation versus $\theta$ decreases for rods and increases for platelets. The solution profiles switch concavity in the boundary layer for flow-aligning versus tumbling discotics, whereas the concavity remains the same for rods. Figure 7 depicts typical steady solutions for tumbling rods and discs. 
TABLE 2

Steady-state features of the order parameter morphology for Couette flow with normal anchoring.

\begin{tabular}{|c|c|c|c|c|}
\hline & FA/rods & FA/discs & T/rods & T/discs \\
\hline$s-s_{0}$ & Concave down & $\begin{array}{c}\text { Concave down } \\
\text { \& Concave up in BL }\end{array}$ & $\begin{array}{c}\text { Concave down } \\
\text { \& Concave up in BL }\end{array}$ & $\begin{array}{c}\text { Concave up } \\
\text { \& Concave down in BL }\end{array}$ \\
\hline$\beta$ & Concave up & Concave up & $\begin{array}{c}\text { Concave up } \\
\text { \& Concave down in BL }\end{array}$ & Concave down \\
\hline
\end{tabular}

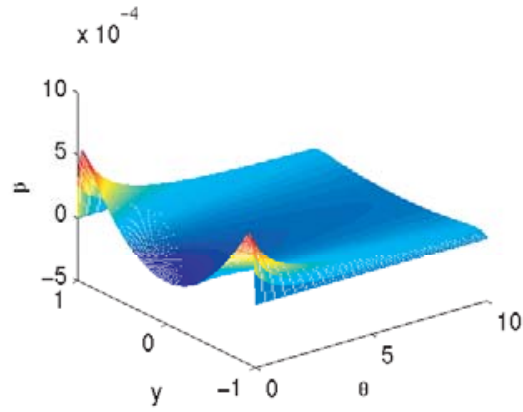

(a)

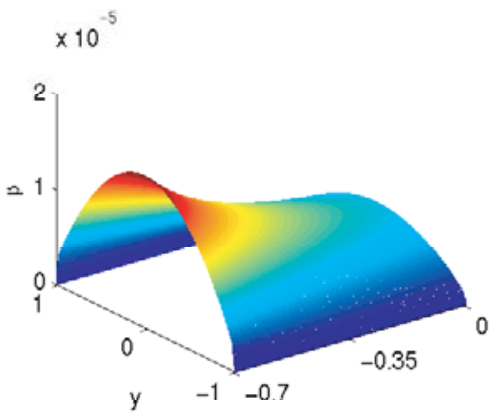

(c)

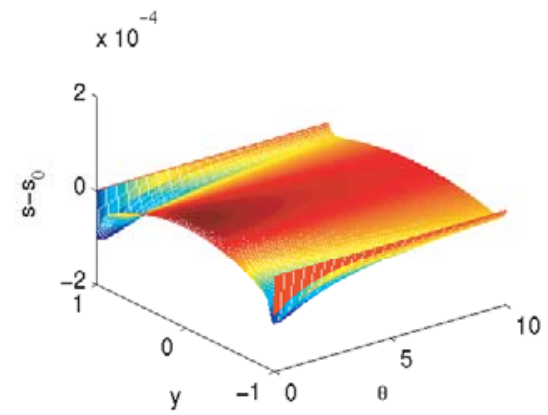

(b)

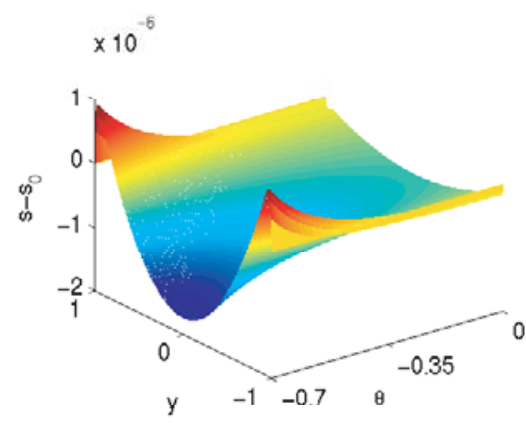

(d)

FIG. 7. The steady-state asymptotic solution as functions of $(\theta, y)$ in tumbling regime with normally anchored boundary condition in weak shear. (a) and (b) depict the solution for rods with parameter values $a=0.8, N=6, D e=0.01, E r=100$. (c) and (d) depict the solution for discs with parameter values $a=-0.8, N=6, D e=0.01, E r=100$.

Again the governing system can become ill-posed. The growth rate formulae for the steady states are identical to (41), but with new coefficients. Notice that the discriminant $A_{1} B_{2}-A_{2} B_{1}=4\left(1-s_{0}\right)^{2}\left(1+2 s_{0}\right)\left[3+\theta\left(1+2 s_{0}\right)\right]\left[3+\theta\left(1+4 s_{0}\right)\right]$. For rods, $\theta>0$ and $A_{1} B_{2}-A_{2} B_{1}>0$ indicating stability. For discs and $\theta<-\frac{3}{1+4 s_{0}}$, the discriminant is negative so that the steady state is unstable.

Proposition 4. The system for the two order parameters $\left(s_{2}, \beta_{2}\right)$ is locally illposed if and only if $\theta<\theta_{d}$, where

$$
\theta_{d}=-\frac{3}{1+4 s_{0}} .
$$

The shear viscosity at the walls in this case is given by

$$
\eta_{\text {wall }}=\frac{\tau_{x y}}{\frac{d v_{x}^{(1)}}{d y}}=\frac{\alpha a^{2}\left(1+\frac{1}{\lambda_{L}}\right)\left(2 \theta\left(2 s_{0}+1\right)+3\left(1+\frac{1}{\lambda_{L}}\right)\right)}{36\left(\theta\left(2 s_{0}+1\right)+3\right)}+\frac{1}{3}\left(\mu_{1} s_{0}+3 \eta\right) .
$$



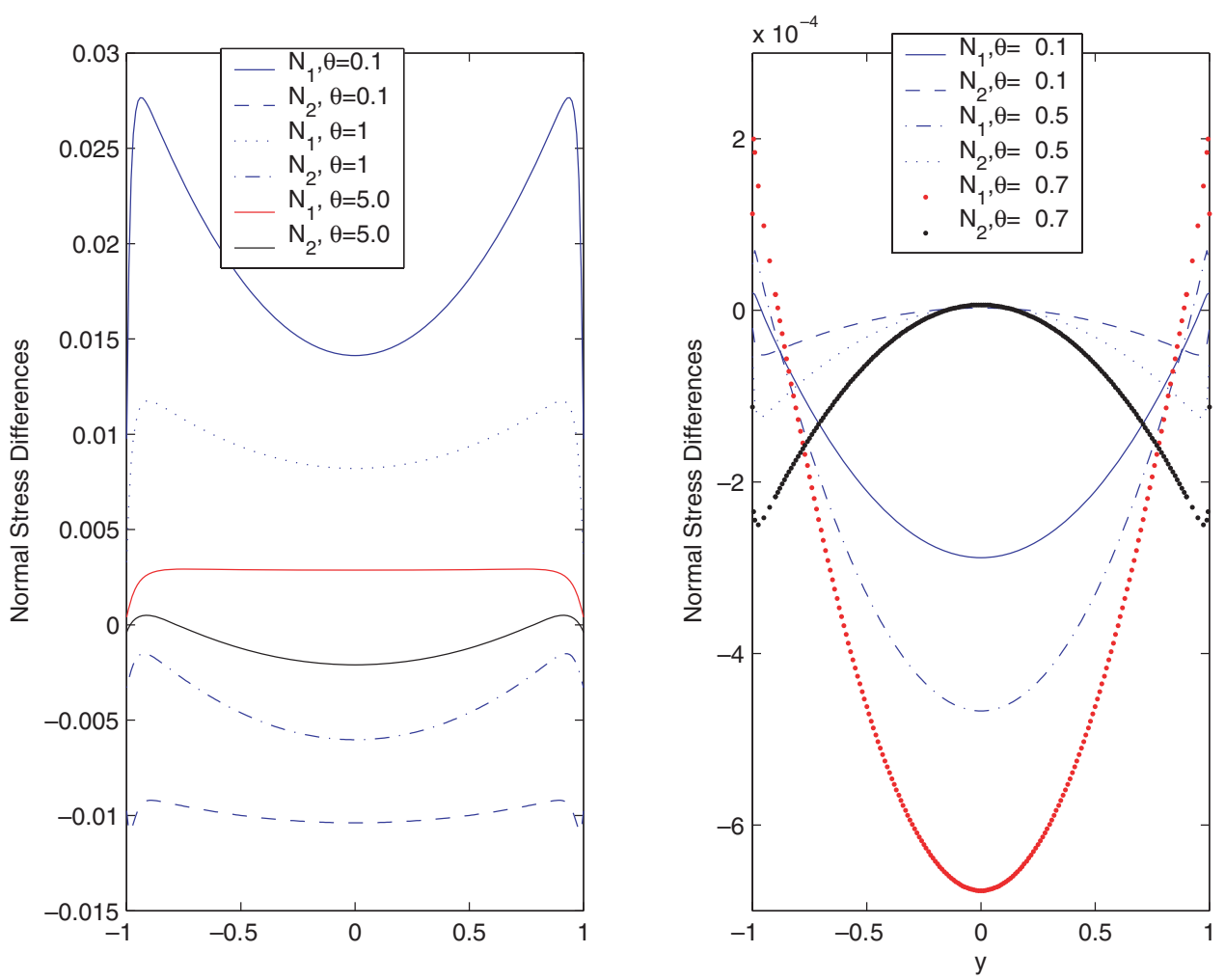

FIG. 8. The normal stress differences $N_{1}$ and $N_{2}$ as functions of $y$ in tumbling regime at selected values of $\theta$ with normally anchored boundary conditions in weak shear. The parameter values are $a= \pm 0.8, N=6, D e=0.01, E r=100, \alpha=10, \mu_{1}= \pm 0.01, \mu_{2}=0.02$ for rods and discs, respectively.

Similar to the case of tangential anchoring, the viscosity decreases with respect to $\theta$ for rods and flow-aligning discs, but increases with respect to $\theta$ for tumbling discs. The leading order normal stress differences again show up at $O\left(D e^{2}\right)$ and are given in Appendix D.

Figures 8 and 9 depict representative plots of the first and second normal stress differences in tumbling and flow-aligning regimes, respectively, and demonstrate the following properties:

- Rods are more sensitive to the variation in degree $\theta$ of elastic anisotropy than discs.

- For tumbling rods, $N_{1}>0$ and $N_{2}<0$ except there may be a sign change in a boundary layer near the wall at large $\theta$. For tumbling discs, $N_{1}$ and $N_{2}$ are both negative except that $N_{1}$ is positive in a boundary layer near the wall.

- The behavior of the normal stress differences does not change much for flowaligning rods compared with tumbling rods. For flow-aligning discs, $N_{1}>0$ and $N_{2}>0$ except that $N_{2}$ is negative in boundary layer near the wall.

- The average values across the gap obey $N_{1}>0$ and $N_{2}<0$ for rods, but they may change signs for discs.

The averaged normal stress differences are tabulated in Table 3.

In summary, the salient predictions from this analysis are: 

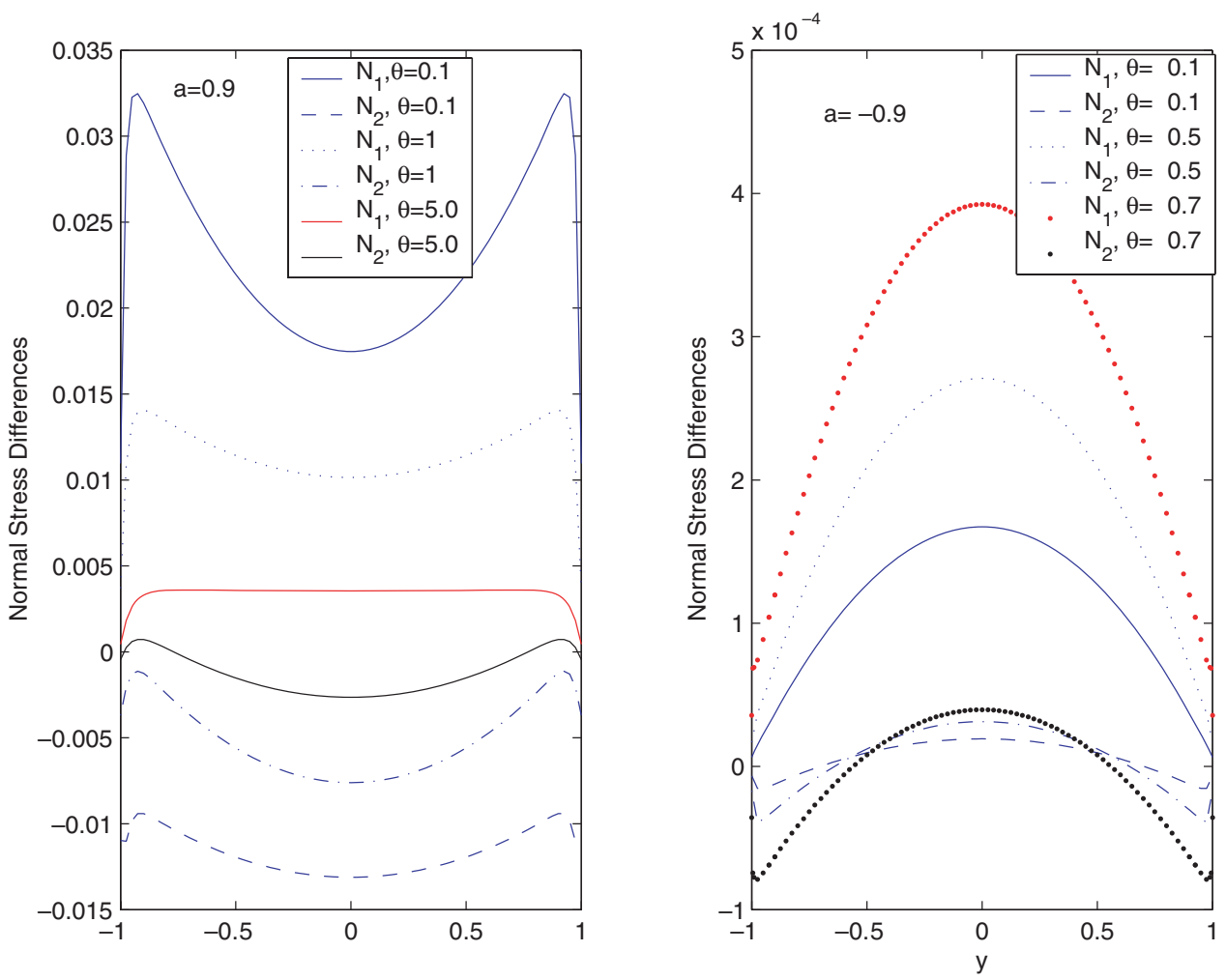

FIG. 9. The normal stress differences $N_{1}$ and $N_{2}$ as functions of $y$ in flowing-aligning regime at selected values of $\theta$ with normally anchored boundary conditions in weak shear. The parameter values are $a= \pm 0.9, N=6, D e=0.01, E r=100, \alpha=10, \mu_{1}= \pm 0.01, \mu_{2}=0.02$ for rods and discs, respectively.

TABLE 3

The averaged normal stress differences (Couette).

\begin{tabular}{|c|c|c|c|c|}
\hline & FA/rods & FA/discs & T/rods & T/discs \\
\hline Tangential & $N_{1}<0, N_{2}>0$ & $N_{1}>0, N_{2}<0$ & $N_{1}>0, N_{2}<0$ & $N_{1}>0, N_{2}<0$ \\
\hline Normal & $N_{1}>0, N_{2}<0$ & $N_{1}>0$ & $N_{1}>0, N_{2}<0$ & $N_{1}<0, N_{2}<0$ \\
\hline
\end{tabular}

- The major director winds counterclockwise from the bottom to top plates, for both flow-aligning rods in tangential anchoring and flow-aligning discs in homeotropic anchoring. Remarkably, the principal orientation axis rotates clockwise if the nematic polymer tumbles in weak shear rather than flow aligns. The magnitude of winding of the orientation axis, which sets the number of bands of nematic distortion, reduces with the degree of elastic anisotropy $\theta$.

- The order parameters are relatively insensitive to the degree of elastic anisotropy in tangential anchoring, and more sensitive in normal anchoring.

- Ill-posedness may occur within each order of asymptotic equations depending on the values of the parameters, although the full equations are well posed. This transition implies a breakdown in the asymptotic ordering which allows explicit solution and scaling properties, and suggests a physical transition away from these asymptotic structures. 


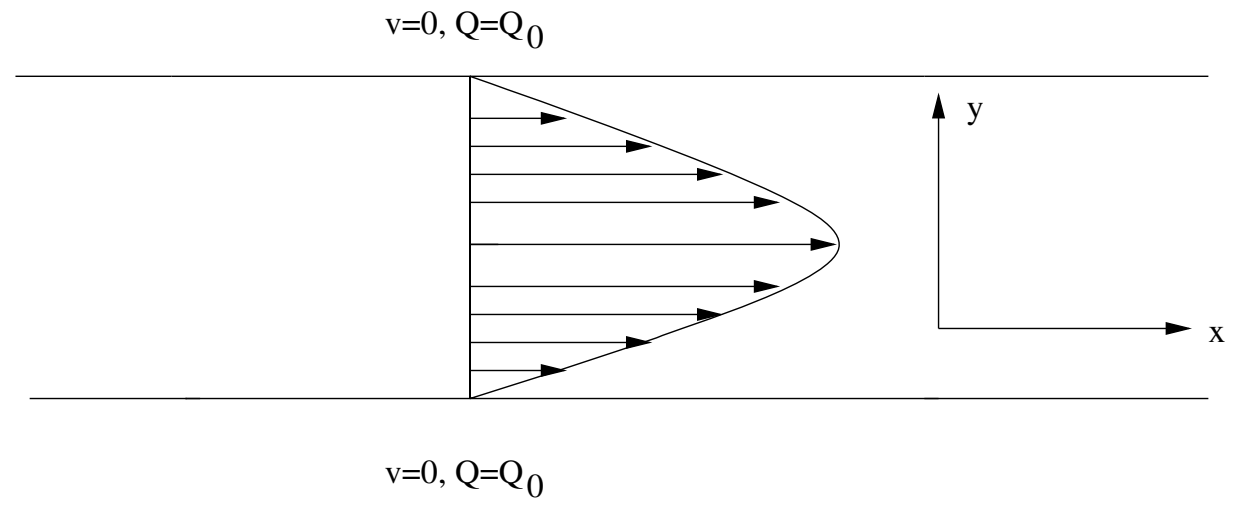

FIG. 10. The geometry of the plane Poiseuille flow. A pressure gradient $\frac{\partial p}{\partial x}=-D e^{2}$ is imposed across the channel. At the bounding surfaces, the orientation tensor is assumed to equal to its equilibrium value.

- The averaged shear viscosity varies weakly with respect to the strength of anisotropic elasticity.

- The averaged normal stress differences may take on all possible signs depending on the parameter regime.

3. Spatial structures in weak Poiseuille flows. In this section, we study steady structures in the direction of the velocity gradient under an imposed, small pressure gradient $\frac{\partial p}{\partial x}$ in plane Poiseuille flow. Figure 10 depicts the cross section of the plane Poiseuille flow on the $(x, y)$ plane. The boundary condition for the orientation tensor is identical to that used for shear flows while the velocity boundary condition is no-slip $\mathbf{v}( \pm 1)=\mathbf{0}$. As before, we use $h$ and $t_{0}=t_{n}=\frac{1}{D_{r}}$ as the characteristic length and time scale, respectively. We adopt the same dimensionless symbols used in weak plane Couette and assume $\frac{\partial p}{\partial x}=-\epsilon=-D e^{2}$ in the dimensionless form, where the Deborah number is defined by

$$
D e=\sqrt{-\frac{\partial p}{\partial x} \frac{t_{0}^{2}}{\rho h}}, \quad \epsilon=D e^{2} .
$$

We seek asymptotic solutions in powers of $\epsilon$. The momentum equation yields at order $O(\epsilon)$ :

$$
\frac{\partial v_{x}^{(1)}}{\partial t}=-1+\frac{\partial \tau_{x y}^{(1)}}{\partial y} .
$$

The other governing equations are identical to those derived for plane Couette flows. Hence, the transient solution and the stability of steady states are identical to the corresponding problems in plane Couette flows. We will not repeat them here; instead, we only present the asymptotic steady states with respect to the two anchoring conditions.

3.1. Tangential anchoring $\left(\psi_{0}=\mathbf{0}\right)$. The steady solutions up to order $O(\epsilon)$ are

$$
s_{0}=s_{0}, \quad \beta_{0}=0, \quad v_{x}^{(1)}(y)=H_{1}\left(1-y^{2}\right), \quad \psi^{(1)}(y)=H_{2} \operatorname{Ery}\left(1-y^{2}\right)
$$


where $H_{1}$ and $H_{2}$ are given in Appendix E. The positivity of $H_{1}$ coincides with the stability of the steady state, giving rise to a parabolic velocity profile. $H_{2}$ is positive for flow aligning rods and negative otherwise in stable steady states. $H_{2}$ behaves more or less like the diagnostic $M$ in the plane Couette flow. Notice that $\psi^{(1)}$ is an odd function of $y$ leading to an asymmetric major director pattern, known as a chevron pattern, with respect to the midplane $[6,2,3]$.

For flow-aligning rods in stable steady states, $H_{1}\left(H_{2}\right)$ decreases (increases) with respect to $\theta$, and increases (decreases) with respect to $\theta$ in all other cases. The rotational pattern of the major director (a function of $\psi^{(1)}$ ) is dictated by $\lambda_{L}$. For flow-aligning rods, $\lambda_{L}>1$, the major director rotates counterclockwise from the lower plate to the $\frac{\sqrt{3}}{6}$ of the shear cell and then reverses its rotation to the midplane. The orientation pattern in the top half of the cell is the mirror image of that in the lower half. The rotation reverses for the other cases where $\lambda_{L}<1$.

The steady solutions of the order parameters at order $O\left(\epsilon^{2}\right)$ are given by

$$
\begin{aligned}
& \beta_{2}(y)=K_{11}\left(\frac{\cosh (\sqrt{E r} \Lambda y)}{\cosh (\sqrt{E r} \Lambda)}-1\right)+K_{12}\left(\frac{\cosh (\sqrt{E r} \Gamma y)}{\cosh (\sqrt{E r} \Gamma)}-1\right)+R_{11}\left(y^{4}-1\right)+R_{12}\left(y^{2}-1\right), \\
& s_{2}(y)=K_{21}\left(\frac{\cosh (\sqrt{E r} \Lambda y)}{\cosh (\sqrt{E r} \Lambda)}-1\right)+K_{22}\left(\frac{\cosh (\sqrt{E r} \Gamma y)}{\cosh (\sqrt{E r} \Gamma)}-1\right)+R_{21}\left(y^{4}-1\right)+R_{22}\left(y^{2}-1\right),
\end{aligned}
$$

where the coefficients are given in Appendix E. The order parameters behave like a quartic polynomial with respect to $y$ in most part of the cell except at the boundary layers near the plates. The velocity $v_{x}^{(1)}$, angle variable $\psi^{(1)}$ and the order parameter $\beta_{2}$ are insensitive to the variation of $\theta$. The sensitivity of the order parameter correction $s_{2}$ is the most pronounced at $\theta=0$, i.e., in the one-constant approximation. Figure 11 depicts typical steady solutions for tumbling rods. Table 4 tabulates all the steady state behavior:

- The thickness of the boundary layers in this flow are narrower than those in the weak plane Couette flows, suggesting a mollifying effect of stronger velocity gradients near the walls.

- The two order parameter corrections (at small $|\theta|$ ) and the angle variable change their signs and concavity in the tumbling versus flow-aligning regime, but only for rods; this predicts a profile concavity flip in the focusing and defocusing of the orientation distribution occurs as rods pass through the flow-aligning to tumbling transition; the profile of $\beta_{2}$ is either $\mathrm{W}$-shaped or M-shaped.

- The velocity profile is concave down.

- The parameter $s-s_{0}$ is very sensitive around $\theta=0$ for both rods and discs.

- The angle profile is a rotated-S shape.

Figure 11 depicts typical steady solutions for tumbling discs as functions of $(\theta, y)$.

The shear viscosity is given by

$$
\eta_{a p p}=\frac{\tau_{x y}}{\frac{d v_{x}^{(1)}}{d y}}=-\frac{\alpha H_{2} s_{0}}{18 H_{1}}\left[a\left(s_{0}+2\right)\left(\theta\left(1-s_{0}\right)+3\left(1-\frac{1}{\lambda_{L}}\right)\right)\right]+\frac{1}{3}\left(\mu_{1} s_{0}+3 \eta\right)
$$

a decreasing function with respect to $\theta$ for discs and tumbling rods but an increasing function for flow-aligning rods.

The normal stress differences in this case are given in Appendix E. Unlike weak Couette flows, they are rational functions of $\mu_{1}, \mu_{2}$ and $\alpha$. Figures 12 and 13 


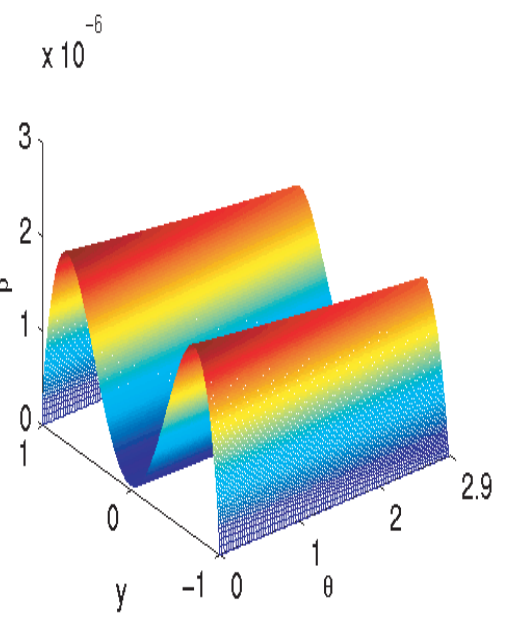

(a)

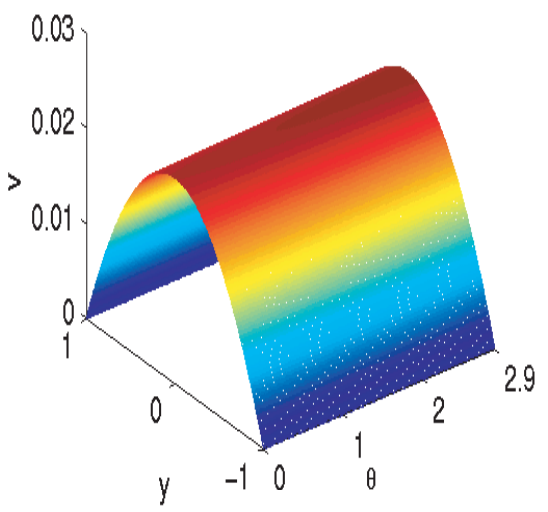

(c)

$$
\times 10^{-5}
$$

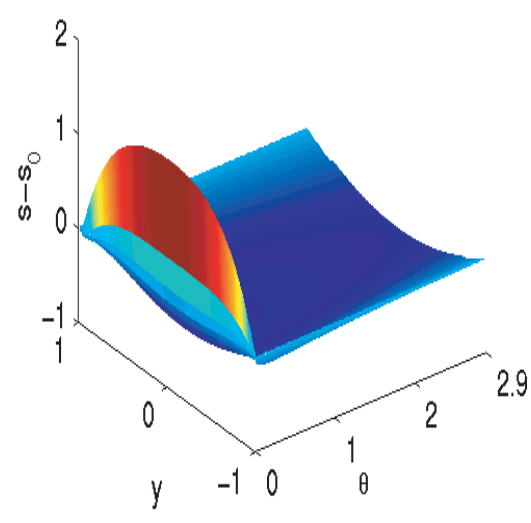

(b)

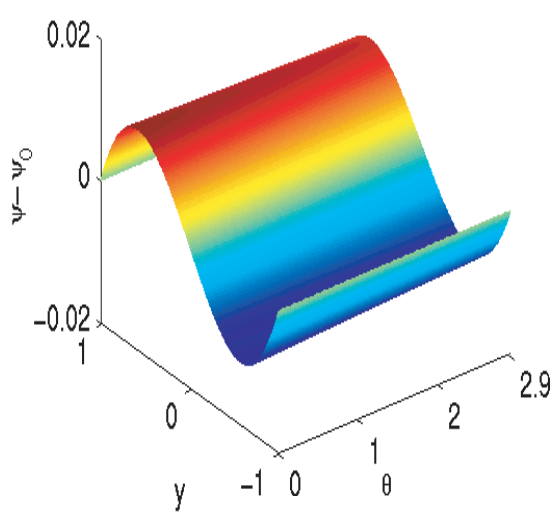

(d)

FIG. 11. Steady solutions of $\beta, s-s_{0}, v$ and $\psi-\psi_{0}$ as functions of $(\theta, y)$ in the regime of tumbling rods with tangentially anchored boundary conditions in plane Poiseuille flow. The parameter values are $N=6, a=0.8, D e=0.01, E r=100, \mu_{1}=0.1, \eta=0.2, \alpha=10$.

depict some representative plots of the normal stress differences for tumbling and flow-aligning nematics, respectively. In summary, they have the following properties:

- For flow-aligning rods, $N_{1}$ is positive and $N_{2}$ is negative. The signs are reversed for flow-aligning discs.

- For tumbling rods and discs, $N_{1}$ is negative, but $N_{2}$ is positive.

- In both stable tumbling and flow-aligning regimes, for rods and discs, the absolute values of $N_{1}$ and $N_{2}$ increase and decrease, respectively, as $\alpha$ increases. 
TABLE 4

Steady states (Poiseuille) in tangential anchoring.

\begin{tabular}{|c|c|c|c|c|}
\hline$s-s_{0}$ & FA/rods & FA/discs & T/rods & T/discs \\
\hline$\beta$ & W-shape & M-shape & $\begin{array}{l}\text { Concave down at small } \theta \\
\text { \& Concave up at large } \theta\end{array}$ & Concave up \\
\hline$\psi-\psi_{0}$ & $\mathrm{~S}$ & Rotated-S & Rotated-S & R-shape \\
\hline
\end{tabular}
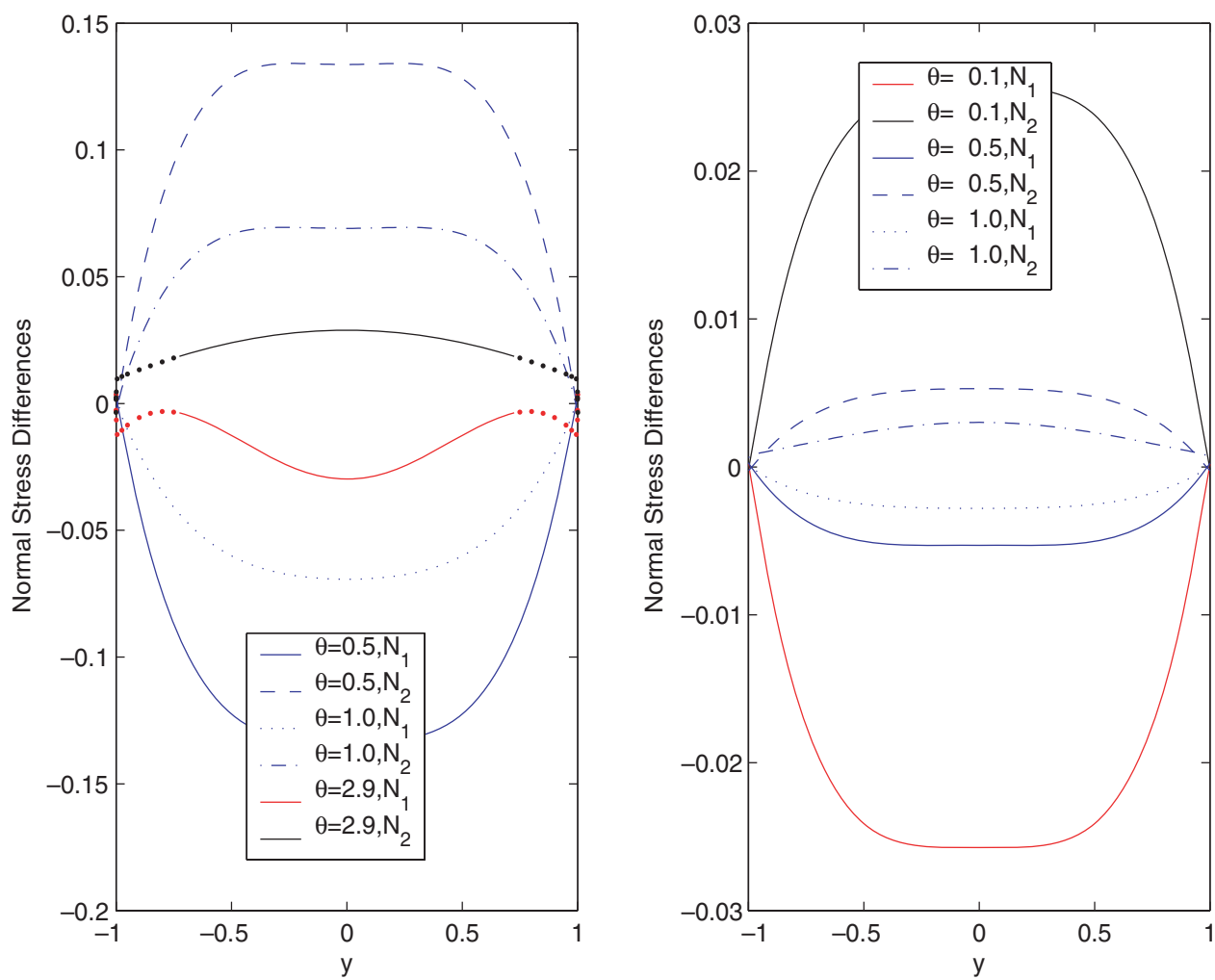

FIG. 12. The normal stress differences $N_{1}$ and $N_{2}$ as functions of $y$ in tumbling regime at selected values of $\theta$ with tangentially anchored boundary conditions in plane Poiseuille flows. The other parameter values are $N=6, D e=0.01, E r=100, \alpha=10, \mu_{2}=0.02$, for rods $a=0.8, \mu_{1}=$ 0.01 , and for discs, $a=-0.8, \mu_{1}=-0.01$.

- The gap averages satisfy $N_{1}>0$ and $N_{2}<0$ for flow-aligning rods while $N_{1}<0, N_{2}>0$ in all other regimes.

The behavior of the averaged normal stress differences is tabulated in Table 6 .

3.2. Homeotropic anchoring $\left(\psi=\frac{\pi}{2}\right)$. The steady solutions up to order $O(\epsilon)$ are given by (57) with new $H_{1}$ and $H_{2}$ given in Appendix F. As in the tangential anchoring case, $H_{1}$ is positive in all stable steady states. $H_{2}$ is negative for flowaligning discs and positive otherwise for other stable steady states. For flow-aligning discs in stable steady states, $H_{1}\left(H_{2}\right)$ decreases (increases) with respect to $\theta$ for flowaligning discs, yet increases (decreases) with respect to $\theta$ for all other stable steady states. 

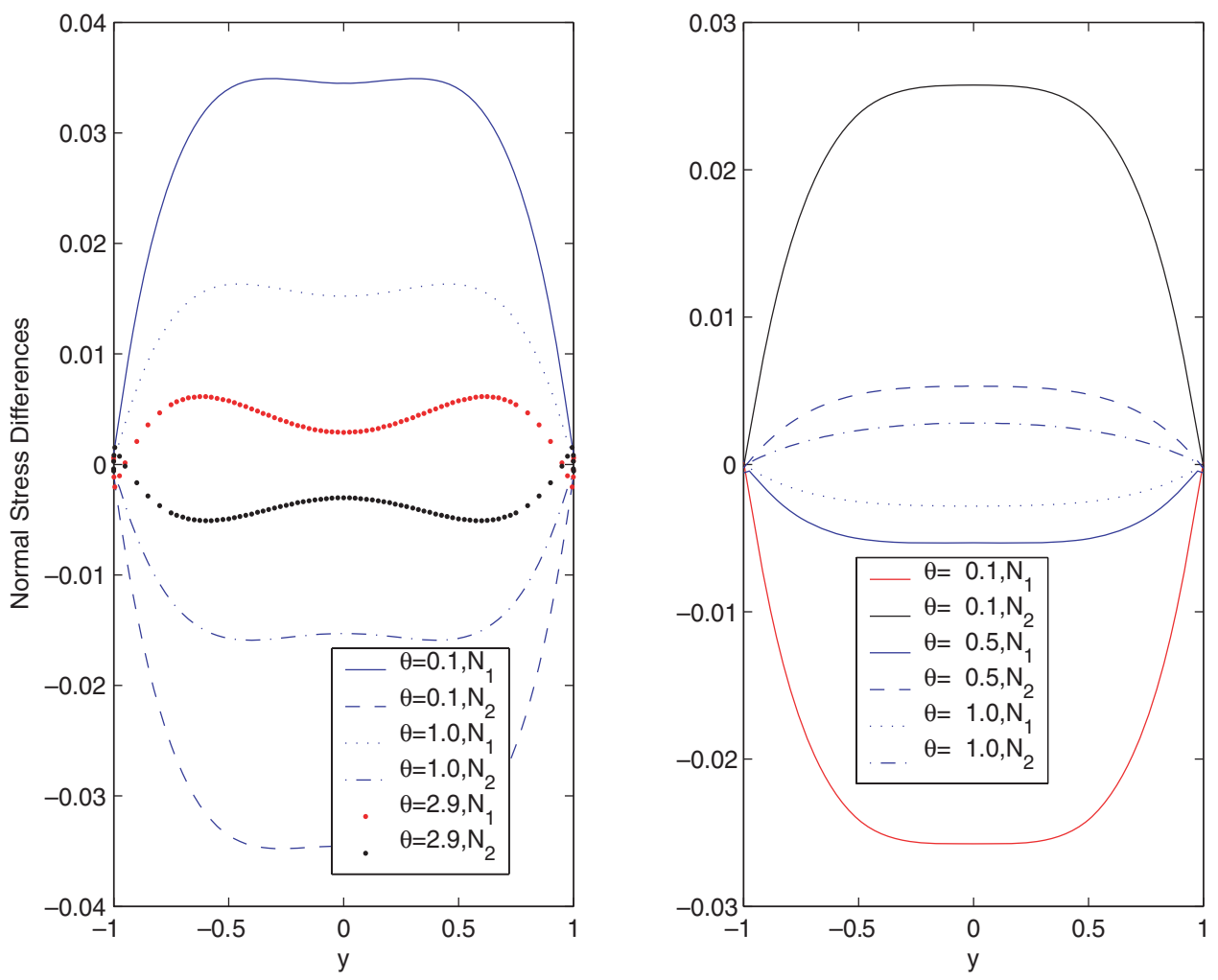

FIG. 13. The normal stress differences $N_{1}$ and $N_{2}$ as functions of $y$ in flow-aligning regime at some values of $\theta$ with tangentially anchored boundary conditions in plane Poiseuille flows. The other parameter values are $N=6, D e=0.01, E r=100, \alpha=10, \mu_{2}=0.02$, for rods $a=0.9, \mu_{1}=0.01$, and for discs, $a=-0.9, \mu_{1}=-0.01$.

TABLE 5

Steady states (Poiseuille) in homeotropic anchoring.

\begin{tabular}{|c|c|c|c|c|}
\hline & FA/rods & FA/discs & T/rods & T/discs \\
\hline$s-s_{0}$ & W-shape & M-shape & W-shape & W-shape \\
\hline$\beta$ & W-shape & M-shape & W-shape & W-shape \\
\hline$\psi-\psi_{0}$ & Rotated-S & S & Rotated-S & Rotated-S \\
\hline
\end{tabular}

The steady solutions of the order parameters at $O\left(\epsilon^{2}\right)$ are

$$
\begin{aligned}
\beta_{2}(y)= & K_{11}\left(\frac{\cosh (\sqrt{E r} \Lambda y)}{\cosh (\sqrt{E r} \Lambda)}-1\right)+R_{1}\left(y^{4}-1\right)+S_{1}\left(y^{2}-1\right), \\
s_{2}(y)= & K_{21}\left(\frac{\cosh (\sqrt{E r} \Lambda y)}{\cosh (\sqrt{E r} \Lambda)}-1\right) \\
& +K_{22}\left(\frac{\cosh (\sqrt{E r} \Gamma y)}{\cosh (\sqrt{E r} \Gamma)}-1\right)+R_{2}\left(y^{4}-1\right)+S_{2}\left(y^{2}-1\right) .
\end{aligned}
$$

We summarize the features of stable steady states in Table 5:

- The two order parameters and the angle parameter change their signs and shapes in tumbling and flow-aligning regime for discs but not for rods, indicating the solutions are more sensitive for platelet molecules than for rods. 


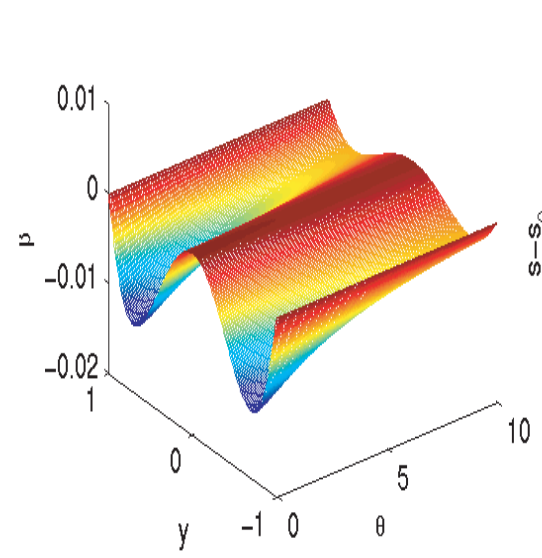

(a)

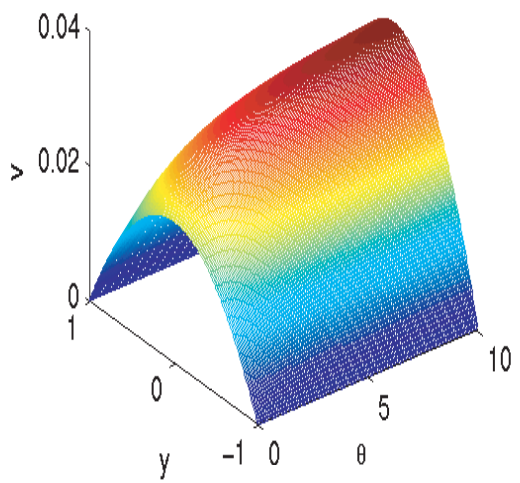

(c)

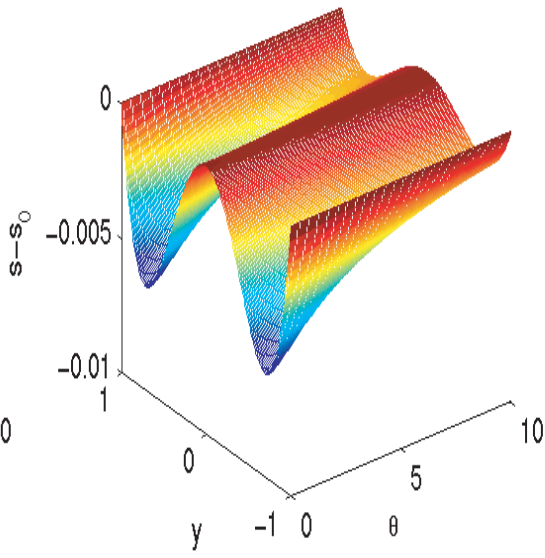

(b)

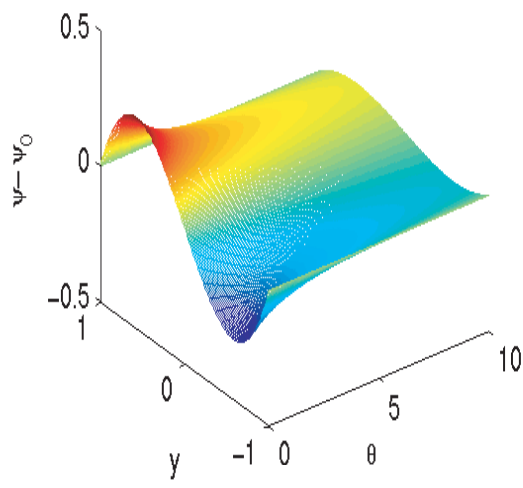

(d)

FIG. 14. Steady solutions $\beta, s-s_{0}, v$ and $\psi-\psi_{0}$ for tumbling rods as functions of $(\theta, y)$ with normally anchored boundary conditions in plane Poiseuille flows. The parameter values are $a=0.8, N=6, D e=0.01, E r=100, \mu_{1}=0.01, \eta=0.02, \alpha=1$.

- As the anisotropic elasticity enhances, the order parameter variations and the angle variation reduces for rods yet amplifies for discs.

- The velocity profile has fixed concavity in all regimes. As the anisotropic elasticity increases, the velocity increases for rods.

- The orientational variables decrease with respect to $\theta$ while the flow variable $v$ increases.

- Again, the thickness of the boundary layers are smaller compared to weak plane Couette flows.

Figure 14 depicts a typical steady solution for tumbling discs as functions of $(\theta, y)$. 

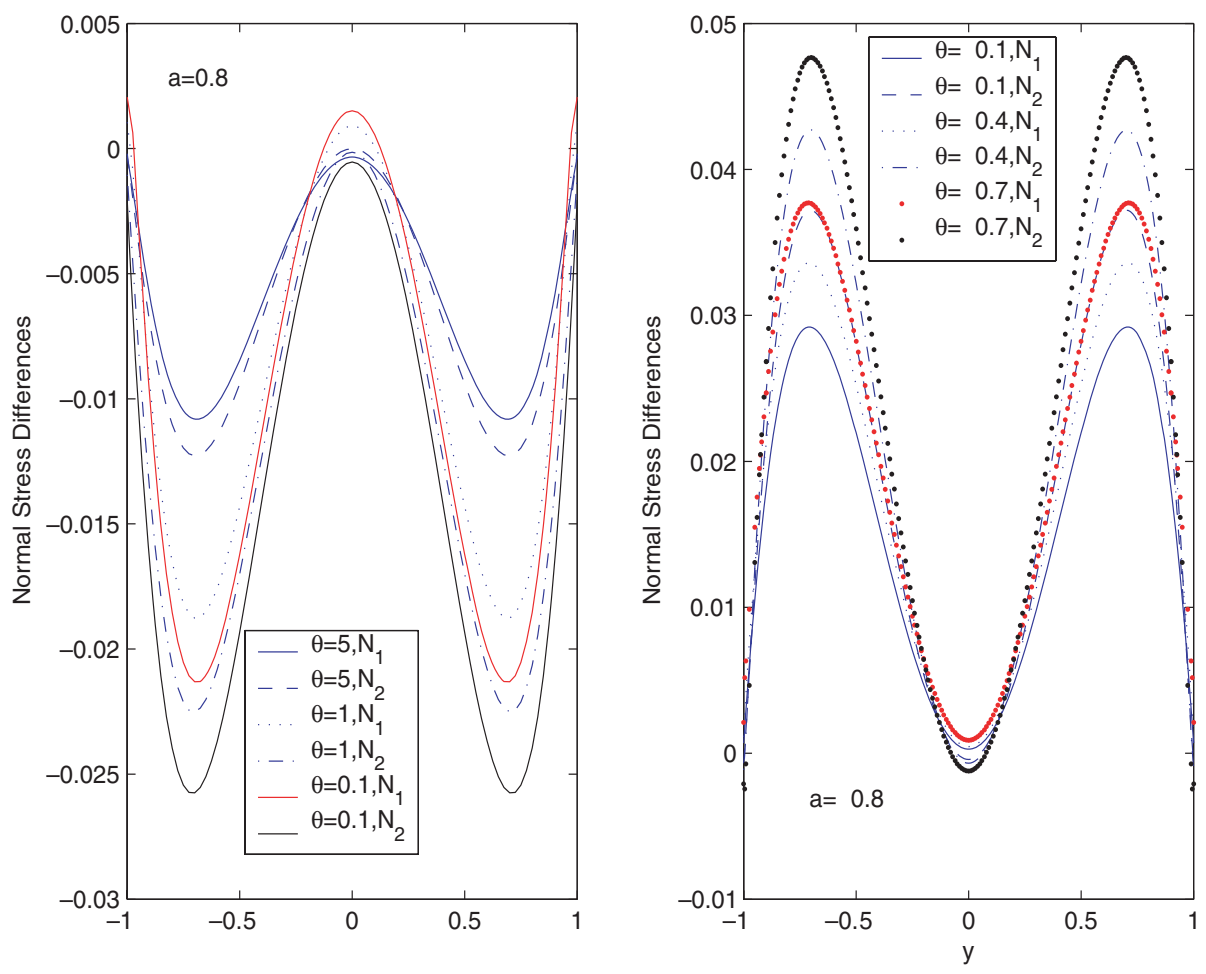

FIG. 15. The normal stress differences $N_{1}$ and $N_{2}$ as functions of $y$ in tumbling regime at selected values of $\theta$ with normal anchoring boundary conditions in plane Poiseuille flows. The parameter values are $N=6, D e=0.01, E r=100, \alpha=10, a=0.8, \mu_{1}=0.01, \mu_{2}=0.02$ for rods, and $a=-0.8, \mu_{1}=-0.01, \mu_{2}=0.02$ for discs.

The wall shear viscosity is given by

$$
\eta_{\text {wall }}=\frac{\tau_{x y}}{\frac{d v_{x}^{(1)}}{d y}}=\frac{\alpha H_{2}}{18 E r H_{1}}\left(a\left(2+s_{0}\right)\left(\theta\left(2 s_{0}+1\right)+3\left(1+\frac{1}{\lambda_{L}}\right)\right)\right)+\frac{1}{3}\left(\mu_{1} s_{0}+3 \eta\right),
$$

which decays with respect to $\theta$ for all rods and flow-aligning discs, but increases for tumbling discs. The first and second normal stress differences in this case are given in Appendix F. Figures 15 and 16 depict the normal stress differences for flow-aligning and tumbling nematics, respectively. In summary, they exhibit the following features:

- For flow-aligning rods and disks, $N_{1}$ and $N_{2}$ are negative except for a small region at the midplane, where some of the first normal stress difference may be positive.

- For tumbling rods, $N_{1}$ and $N_{2}$ are negative. For tumbling discs $\left(-1<\lambda_{L}<\right.$ $0), N_{1}$ and $N_{2}$ are negative except for a tiny region at the midplane at small $\theta$. For tumbling discs, the behavior reverses completely, i.e., the normal stress differences are positive except for a small region at the midplane.

- In both stable tumbling and flow-aligning regimes, for rods and discs, the absolute values of $N_{1}$ and $N_{2}$ will decrease and increase as $\alpha$ increases, respectively.

- The gap averages obey $N_{1}>0$ and $N_{2}>0$ for tumbling rods and both become negative in all other regimes.

The behavior of averaged normal stress differences is tabulated in Table 6 . 

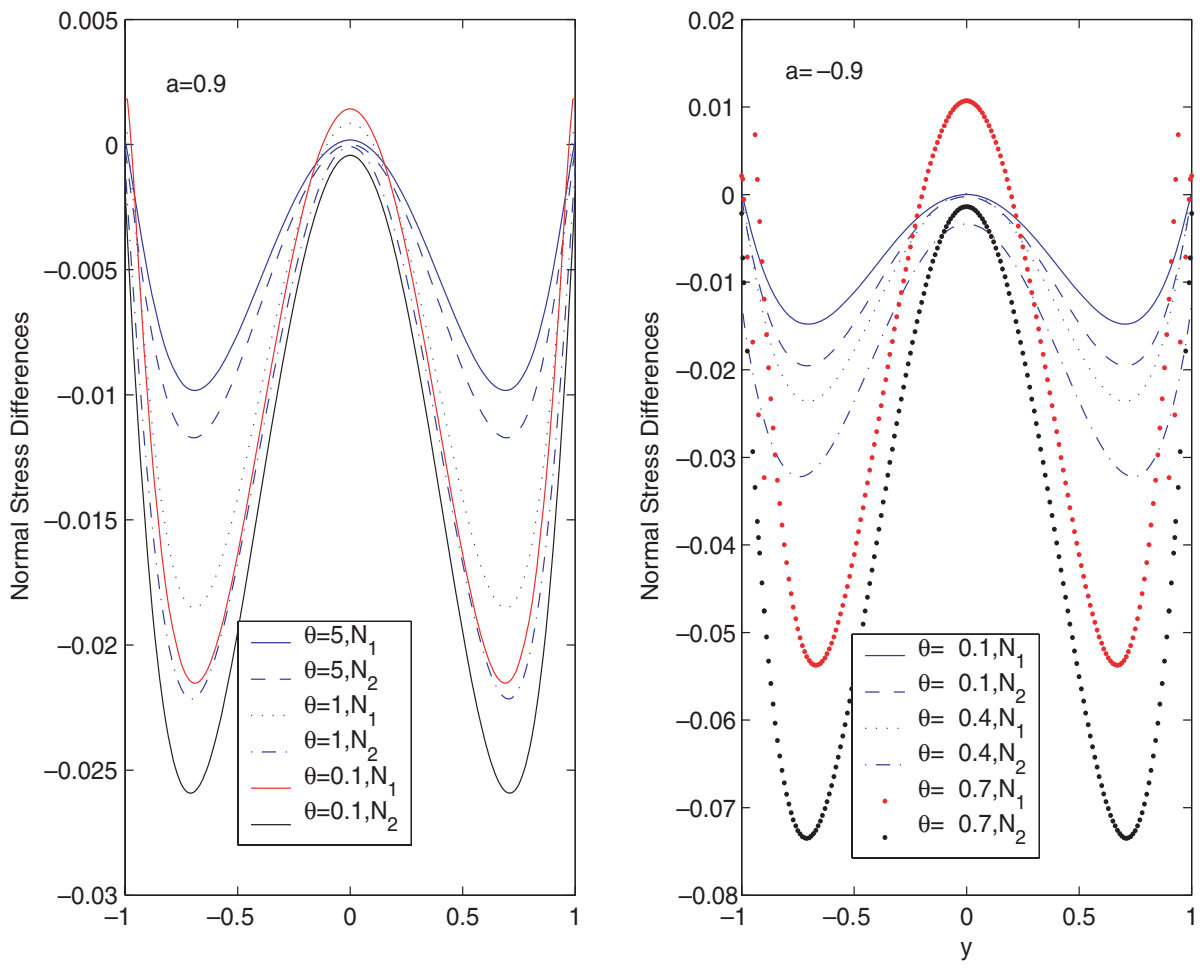

FIG. 16. The normal stress differences $N_{1}$ and $N_{2}$ as functions of $y$ in flow-aligning regime at selected values of $\theta$ with normal anchoring boundary conditions in plane Poiseuille flows. The parameter values are $N=6, D e=0.01, E r=100, \alpha=10, a=0.9, \mu_{1}=0.01, \mu_{2}=0.02$ for rods, and $N=6, D e=0.01, E r=100, \alpha=1, a=-0.9, \mu_{1}=-0.01, \mu_{2}=0.02$ for discs.

TABLE 6

The normal stress differences (Poiseuille).

\begin{tabular}{|c|c|c|c|c|}
\hline & FA/rods & FA/discs & T/rods & T/discs \\
\hline Tangential & $N_{1}>0, N_{2}>0$ & $N_{1}<0, N_{2}>0$ & $N_{1}<0, N_{2}>0$ & $N_{1}<0, N_{2}>0$ \\
\hline Normal & $N_{1}<0, N_{2}<0$ & $N_{1}<0, N_{2}<0$ & $N_{1}<0, N_{2}<0$ & $N_{1}>0, N_{2}>0$ \\
\hline
\end{tabular}

4. Conclusion. We have derived explicit asymptotic structures for weakly sheared nematic polymers in both plate-driven and pressure-driven experimental conditions. The goal of this analysis is to predict scaling properties in the orientational distribution of the rigid rod ensemble from the strong elasticity, weak flow regime, which then guide numerical continuation studies of heterogeneous films and molds across a multi-parameter space of material properties and processing conditions. We have explored the effect of anisotropic elasticity for both flow conditions, using a second-moment model for the orientational distribution derived from Doi-HessMarrucci-Greco kinetic theory. These results extend previous work of the authors for steady structures with equal elasticity constants in plate-driven flow in several ways: anisotropic elasticity, pressure-driven flows, and transient asymptotic equations followed by stability predictions within the asymptotic ordering of the flow-nematic system. The leading order flow structure is simple linear shear versus Poiseuille profiles for the respective driving conditions, with elastic hydrodynamic feedback contributions characterized at next order. These results confirm the consistency of imposing 
the kinematics (and thereby suppressing flow feedback) in special asymptotic parameter regimes, yet also predict breakdown of this decoupling of the momentum equation when either of several conditions is relaxed: weak flow, strong elasticity, or sufficiently isotropic elasticity.

The orientational structures for both flow conditions convey scaling properties of nematic (director dominated) elastic distortions as well as molecular elasticity (dominated by focusing or defocusing of the orientational distribution). The structure scaling laws are similar for plane Couette and Poiseuille flows, with plate boundary layer thicknesses proportional to $1 / \sqrt{E r}$ and nonuniform structures spanning the plates with mean lengthscale proportional to $1 / E r$. The prefactors of the structures capture the roles of material properties: flow-aligning versus tumbling nematics, degree of anisotropy in the elasticity potential, strength of the short-range nematic potential, and molecular aspect ratio. These subtleties are detailed in the body of the paper, where the amplitude of structure variations, convexity of profiles, and stability of the steady structures all depend strongly on these molecular parameters as well as plate anchoring conditions.

The particular results are less important than the overall insight into the sensitivity, flow-nematic feedback and processing-generated structures on material and device properties. The instability within the asymptotic equations is catastrophic, similar to backward heat flow instabilities, so there is no mistaking the breakdown of these steady profiles in the weak flow model system. The role of anisotropic elasticity is shown to be greater for normal versus tangential plate anchoring, and greater in pressure-driven than plate-driven flows. In Poiseuille flow, the transition to catastrophic instability coincides with a rapidly growing midplane axial velocity, confirming a breakdown in the asymptotic analysis. Finally, anisotropic elasticity is shown to contribute to either shear thinning or shear thickening behavior as other parameters are modified, and signs of normal stress differences (which determine whether the plates are pushed or pulled by the stresses generated between) are likewise sensitive to various material parameters.

Appendix A. The coefficients in the second-order equations in tangential anchoring.

$$
\begin{array}{ll}
A_{1} & =\left(1-s_{0}\right)\left[-6\left(1+2 s_{0}\right)+\left(8 s_{0}^{2}-s_{0}-2\right) \theta\right], \quad B_{1}=\left(1-s_{0}\right) s_{0}\left(6-7 s_{0} \theta\right), \\
& C_{1}=18 E r N s_{0}\left(-1+4 s_{0}\right), \quad D_{1}=36 \operatorname{ErN} s_{0}\left(1-s_{0}\right), \\
& E_{1}=2 s_{0}\left(1-s_{0}\right)\left(1+3 s_{0}\right)\left[6+\left(2-5 s_{0}\right) \theta\right], \\
(62) & F_{1}=2 s_{0}\left[3\left(2+s_{0}\right)+2\left(3 s_{0}^{2}+s_{0}-1\right) \theta\right], \quad G_{1}=9 E r s_{0}\left(-1-a+2 a s_{0}\right), \\
& A_{2}=-2 s_{0} \theta\left(1-s_{0}\right), \quad B_{2}=\left(1-s_{0}\right)\left[-6+\left(3 s_{0}-2\right) \theta\right], \quad C_{2}=0, \\
& D_{2}=54 E r N s_{0}, E_{2}=2 s_{0}\left(s_{0}-1\right)[6+(2-5 \theta)], \\
& F_{2}=2 s_{0}\left[3\left(2-s_{0}\right)+\left(s_{0}+2\right)\left(s_{0}-1\right) \theta\right], \quad G_{2}=9 \operatorname{Ers}_{0}(1-a) .
\end{array}
$$

Appendix B. The coefficients in the second-order equations in normal anchoring.

$$
\begin{aligned}
& A_{1}=-2\left(1-s_{0}\right)\left(2 s_{0}+1\right)\left[3+\left(4 s_{0}+1\right) \theta\right], \\
& B_{1}=2 s_{0}\left(1-s_{0}\right)\left[3+2\left(2 s_{0}+1\right) \theta\right], \\
& C_{1}=18 E r N s_{0}\left(-1+4 s_{0}\right), \quad D_{1}=36 E r N s_{0}\left(1-s_{0}\right), \\
& \left.E_{1}=4 s_{0}\left(1-s_{0}\right)\left[3\left(1+3 s_{0}\right)-\left(2 s_{0}+1\right)\left(6 s_{0}+1\right) \theta\right)\right], \\
& F_{1}=2 s_{0}\left[3\left(2+s_{0}\right)-\left(6 s_{0}^{3}-5 s_{0}^{2}-8 s_{0}-2\right) \theta\right], \quad G_{1}=9 \operatorname{Ers}_{0}\left(-1+a-2 a s_{0}\right), \\
& A_{2}=0, \quad B_{2}=2\left(s_{0}-1\right)\left[3+\left(2 s_{0}+1\right) \theta\right], \quad C_{2}=0, \quad D_{2}=54 E r N s_{0}, \\
& E_{2}=4 s_{0}\left[3\left(-1+s_{0}\right)+\left(2 s_{0}^{2}+3 s_{0}-1\right) \theta\right], \\
& F_{2}=2 s_{0}\left[-3\left(2+s_{0}\right)+\left(2 s_{0}+1\right)\left(s_{0}-2\right) \theta\right], \quad G_{2}=9 \operatorname{Ers}_{0}(1+a) .
\end{aligned}
$$


Appendix C. The coefficients of the steady solutions for tangential anchoring in weak plane Couette flows.

$$
\begin{aligned}
& \lambda, \mu=\sqrt{E r}\left\{1 8 \left[N s_{0}\left(s_{0}-1\right)\left(\left(16 s_{0}^{2}-5 s_{0}-2\right) \theta-6\left(5 s_{0}+1\right)\right) \mp\right.\right. \\
& \sqrt{2}\left(\left(68 s_{0}^{4}+49 s_{0}^{3}-24 s_{0}^{2}-20 s_{0}+8\right) \theta^{2}-6\left(8 s_{0}^{3}+21 s_{0}^{2}+6 s_{0}-8\right) \theta+\right. \\
& \left.\left.\left.18\left(s_{0}+2\right)^{2}\right)^{1 / 2} s_{0}\left(1-s_{0}\right) N\right] /\left[\left(s_{0}-1\right)^{2}\left(2 s_{0}+1\right)\left(5 \theta s_{0}-2 \theta-6\right)\left(\theta s_{0}-2 \theta-6\right)\right]\right\}^{\frac{1}{2}}, \\
& R_{1}=\frac{\left(a s_{0}-3 s_{0}+2 a\right)\left(s_{0}-1\right)\left[\left(-5 s_{0}^{2}+2 a s_{0}^{2}+3 a s_{0}+2 s_{0}-2 a\right) \theta-3\left(a s_{0}-2 s_{0}+2 a\right)\right]}{4 N s_{0}^{2}\left(s_{0}+2\right)^{2}\left(\theta s_{0}-\theta-3\right)^{2}} \\
& S_{1}=\left(1-s_{0}\right)\left[\left(-88 s_{0}^{3}+34 a s_{0}+77 a s_{0}^{2}-31 s_{0}^{2}+8 a s_{0}+20 s_{0}-20 a\right) \theta-6\left(7 a s_{0}^{2}-14 s_{0}^{2}\right.\right. \\
& \left.\left.-10 s_{0}+19 a s_{0}+10 a\right)\right]\left(a s_{0}-3 s_{0}+2 a\right) /\left[8 s_{0}^{2} N\left(-1+4 s_{0}\right)\left(s_{0}+2\right)^{2}\left(\theta s_{0}-\theta-3\right)^{2}\right], \\
& \Lambda=\frac{\lambda}{\sqrt{E r}}, \Gamma=\frac{\mu}{\sqrt{E r}}, R=R_{1} E r, S=S_{1} E r, K=\frac{3\left(a s_{0}-3 s_{0}+2 a\right) E r}{4 s_{0}\left(s_{0}+2\right)\left[\left(s_{0}-1\right) \theta-3\right]}, \\
& A=-\left(s_{0}-1\right)^{2}\left(2 s_{0}+1\right)\left(5 \theta s_{0}-2 \theta-6\right)\left(\theta s_{0}-2 \theta-6\right) \text {, } \\
& B=36 E r N s_{0}\left(s_{0}-1\right)\left[\left(16 s_{0}^{2}-5 s_{0}-2\right) \theta-6\left(5 s_{0}+1\right)\right], \quad C=972\left(E r N s_{0}\right)^{2}\left(1-4 s_{0}\right), \\
& T=\frac{2 A_{1}\left(4 K^{2} E_{2}+2 K^{2} F_{2}+K G_{2}\right)-2 A_{2}\left(4 K^{2} E_{1}+2 K^{2} F_{1}+K G_{1}\right)-C_{1}\left(2 K^{2} F_{2}+K G_{2}\right)-2 B R}{C}, \\
& K_{1}=\frac{1}{\lambda^{2}-\mu^{2}}\left[\frac{4 K^{2} A_{1} E_{2}-4 K^{2} A_{2} E_{1}}{A}+\mu^{2}(R+T)-2 R\right], \quad K_{3}=-\frac{B_{2} \lambda^{2}+D_{2}}{A_{2} \lambda^{2}} K_{1}, \\
& K_{2}=\frac{1}{-\lambda^{2}+\mu^{2}}\left[\frac{4 K^{2} A_{1} E_{2}-4 K^{2} A_{2} 4 E_{1}}{A}+\lambda^{2}(R+T)-2 R\right], \quad K_{4}=-\frac{B_{2} \mu^{2}+D_{2}}{A_{2} \mu^{2}} K_{2}, \\
& N_{1}=\frac{G \alpha}{54 E r}\left(\frac{K_{3} \lambda^{2} \cosh (\lambda y)}{\cosh (\lambda)}+\frac{K_{4} \mu^{2} \cosh (\mu y)}{\cosh \mu}+2 S\right) \\
& +\frac{a N s_{0}\left(1-4 s_{0}\right) \alpha}{3}\left[K_{3}\left(\frac{\cosh (\lambda y)}{\cosh (\lambda)}-1\right)+K_{4}\left(\frac{\cosh (\mu y)}{\cosh (\mu)}-1\right)+S\left(y^{2}-1\right)\right] \\
& +\frac{\left(-4 a \theta+3 s_{0}+16 a \theta s_{0}^{3}-12 a+12 a s_{0}^{2}-12 a \theta s_{0}\right) \alpha}{108 E r}\left(\frac{K_{1} \lambda^{2} \cosh (\lambda y)}{\cosh (\lambda)}+\frac{K_{2} \mu^{2} \cosh (\mu y)}{\cosh (\mu)}+2 R\right) \\
& +\frac{N s_{0}\left(1+2 s_{0}\right) a \alpha}{3}\left[K_{1}\left(\frac{\cosh (\lambda y)}{\cosh (\lambda)}-1\right)+K_{2}\left(\frac{\cosh (\mu y)}{\cosh (\mu)}-1\right)+R\left(y^{2}-1\right)\right] \\
& +\frac{4 K^{2} s_{0} \alpha}{27 E r}\left[4 a\left(s_{0}-1\right)\left(2 s_{0}+1\right)\left(3 s_{0}+1\right) \theta-3\left(2 a s_{0}-3 s_{0}-6 a s_{0}^{2}+4 a\right] y^{2}\right. \\
& \left.+\frac{2 a s_{0} K^{2} \alpha}{27 E r}\left[\left(2 s_{0}+1\right)\left(3 s_{0}^{2}-5 s_{0}-4\right)\right) \theta-6\left(s_{0}+2\right)\right]\left(y^{2}-1\right)+2 \mu_{2} K s_{0}^{2}\left(y^{2}-1\right) \text {, } \\
& N_{2}=-\frac{G \alpha}{54 E r}\left(\frac{K_{3} \lambda^{2} \cosh (\lambda y)}{\cosh (\lambda)}+\frac{K_{4} \mu^{2} \cosh (\mu y)}{\cosh \mu}+2 S\right) \\
& +\frac{a N s_{0}\left(-1+4 s_{0}\right) \alpha}{3}\left[K_{3}\left(\frac{\cosh (\lambda y)}{\cosh (\lambda)}-1\right)+K_{4}\left(\frac{\cosh (\mu y)}{\cosh (\mu)}-1\right)+S\left(y^{2}-1\right)\right] \\
& +\frac{\left(8 a \theta+12 a-3+8 a s_{0}-16 a s_{0}^{2}-12 s_{0}\right) s_{0} \alpha}{108 E r}\left(\frac{K_{1} \lambda^{2} \cosh (\lambda y)}{\cosh (\lambda)}+\frac{K_{2} \mu^{2} \cosh (\mu y)}{\cosh (\mu)}+2 R\right) \\
& +\frac{2 N s_{0}\left(1-s_{0}\right) a \alpha}{3}\left[K_{1}\left(\frac{\cosh (\lambda y)}{\cosh (\lambda)}-1\right)+K_{2}\left(\frac{\cosh (\mu y)}{\cosh (\mu)}-1\right)+R\left(y^{2}-1\right)\right] \\
& +\frac{4 K^{2} s_{0} \alpha}{27 E r}\left[-2 a\left(s_{0}-1\right)\left(2 s_{0}+1\right)\left(6 s_{0}+1\right) \theta+3\left(4 a s_{0}-3 s_{0}-6 a s_{0}^{2}+2 a\right)\right] y^{2} \\
& \left.+\frac{2 a s_{0} K^{2} \alpha}{27 E r}\left[-\left(2 s_{0}+1\right)\left(3 s_{0}^{2}-4 s_{0}-2\right)\right) \theta+3\left(s_{0}+2\right)\right]\left(y^{2}-1\right) \\
& -2\left(\mu_{1}+\mu_{2}\right) K s_{0}^{2}\left(y^{2}-1\right), \\
& G=2 a \theta+4 a \theta s_{0}^{2}+6 a s_{0}-3 s_{0}-12 a s_{0}^{2}-16 a \theta s_{0}^{3}+10 a \theta s_{0}+6 a .
\end{aligned}
$$


Appendix D. The coefficients of the steady solutions in normal anchoring in weak plane Couette flows.

$$
\begin{aligned}
& \lambda=\sqrt{E r}\left[\frac{3 N s_{0}}{\left(1-s_{0}\right)\left(2 \theta s_{0}+3+\theta\right)}\right]^{1 / 2}, \mu=\sqrt{E r}\left[\frac{9 N s_{0}\left(-1+4 s_{0}\right)}{\left(1-s_{0}\right)\left(2 s_{0}+1\right)\left(4 \theta s_{0}+3+\theta\right)}\right]^{1 / 2}, \Lambda=\frac{\lambda}{\sqrt{E r}}, \Gamma=\frac{\mu}{\sqrt{E r}}, \\
& R=\frac{9\left(1-s_{0}\right)\left(2 s_{0}+a s_{0}+2 a\right)\left(a s_{0}+3 s_{0}+2 a\right)}{4 N\left(s_{0}+2\right)^{2}\left(2 \theta s_{0}+3+\theta\right) s_{0}^{2}} E r, \quad R_{2}=\frac{R}{E r}, \quad K=\frac{3\left(a s_{0}+3 s_{0}+2 a\right)}{4 s_{0}\left(s_{0}+2\right)\left(2 \theta s_{0}+\theta+3\right)} E r, \\
& T=3\left(-1+s_{0}\right)\left[2\left(2 s_{0}+1\right)\left(s_{0}-1\right)\left(a s_{0}+2 s_{0}+2 a\right) \theta+\operatorname{Er} N s_{0} a\left(s_{0}+2\right)\right. \\
& \left.\left.+6\left(-1+s_{0}\right)\left(2 s_{0}+a s_{0}+2 a\right)\right)\right]\left(a s_{0}+3 s_{0}+2 a\right) /\left[4 N^{2}\left(s_{0}+2\right)^{2}\left(2 \theta s_{0}+3+\theta\right) s_{0}^{3}\right], \\
& S=\left\{3 ( a s _ { 0 } + 3 s _ { 0 } + 2 a ) ( 1 - s _ { 0 } ) \left[\left(2 s_{0}+1\right)\left(-3 s_{0}^{2}+a s_{0}^{2}-4 a s_{0}-12 s_{0}-12 a\right) \theta\right.\right. \\
& \left.\left.+18\left(s_{0}-1\right)\left(a s_{0}+2 s_{0}+2 a\right)\right] /\left[8 N s_{0}^{2}\left(s_{0}+2\right)^{2}\left(2 \theta s_{0}+3+\theta\right)^{2}\left(-1+4 s_{0}\right)\right]\right\} E r, \\
& S_{2}=\frac{S}{E r}, \quad T_{1}=-\frac{A_{1} S+2 B_{1} R+D_{1} T+\left(-2 K^{2} F_{1}-G_{1} K\right)}{C_{1}}, \\
& K_{11}=-R-T, \quad K_{21}=\frac{B_{1} \lambda^{2}+D_{1}}{A_{1} \lambda^{2}+C_{1}} K_{11}, \quad K_{22}=-K_{21}-S-T_{1}, \\
& N_{1}=\frac{G \alpha}{54 E r}\left(\frac{K_{21} \lambda^{2} \cosh (\lambda y)}{\cosh (\lambda)}+\frac{K_{22} \mu^{2} \cosh (\mu y)}{\cosh \mu}+2 S\right) \\
& +\frac{a N s_{0}\left(1-4 s_{0}\right) \alpha}{3}\left[K_{21}\left(\frac{\cosh (\lambda y)}{\cosh (\lambda)}-1\right)+K_{22}\left(\frac{\cosh (\mu y)}{\cosh (\mu)}-1\right)+S\left(y^{2}-1\right)\right] \\
& +\frac{\left(-4 a \theta+3 s_{0}+16 a \theta s_{0}^{3}-12 a+12 a s_{0}^{2}-12 a \theta s_{0}\right) \alpha}{108 E r}\left(\frac{K_{11} \lambda^{2} \cosh (\lambda y)}{\cosh (\lambda)}+2 R\right) \\
& +\frac{N s_{0}\left(1+2 s_{0}\right) a \alpha}{3}\left[K_{11}\left(\frac{\cosh (\lambda y)}{\cosh (\lambda)}-1\right)+R\left(y^{2}-1\right)\right] \\
& +\frac{4 K^{2} s_{0} \alpha}{27 E r}\left[4 a\left(s_{0}-1\right)\left(2 s_{0}+1\right)\left(3 s_{0}+1\right) \theta+3\left(-2 a s_{0}+3 s_{0}+6 a s_{0}^{2}-4 a\right)\right] y^{2} \\
& +\frac{2 a s_{0} K^{2} \alpha}{27 E r}\left[\left(2 s_{0}+1\right)\left(3 s_{0}^{2}-5 s_{0}-4\right) \theta-6\left(s_{0}+2\right)\right]\left(y^{2}-1\right)+2 \mu_{2} K s_{0}^{2}\left(y^{2}-1\right), \\
& N_{2}=-\frac{G \alpha}{54 E r}\left(\frac{K_{21} \lambda^{2} \cosh \lambda y}{\cosh (\lambda)}+\frac{K_{22} \mu^{2} \cosh \mu y}{\cosh \mu}+2 S\right) \\
& +\frac{a N s_{0}\left(-1+4 s_{0}\right) \alpha}{3}\left[K_{21}\left(\frac{\cosh (\lambda y)}{\cosh (\lambda)}-1\right)+K_{22}\left(\frac{\cosh (\mu y)}{\cosh (\mu)}-1\right)+S\left(y^{2}-1\right)\right] \\
& +\frac{\left(8 a \theta+12 a-3+8 a s_{0}-16 a s_{0}^{2}-12 s_{0}\right) s_{0} \alpha}{108 E r}\left(\frac{K_{11} \lambda^{2} \cosh (\lambda y)}{\cosh (\lambda)}+2 R\right) \\
& +\frac{2 N s_{0}\left(1-s_{0}\right) a \alpha}{3}\left[K_{11}\left(\frac{\cosh (\lambda y)}{\cosh (\lambda)}-1\right)+R\left(y^{2}-1\right)\right] \\
& +\frac{4 K^{2} s_{0} \alpha}{27 E r}\left[2 a\left(1-s_{0}\right)\left(2 s_{0}+1\right)^{2}\right) \theta+3\left(4 a s_{0}-9 s_{0}-6 a s_{0}^{2}+2 a\right) y^{2} \\
& +\frac{2 a s_{0} K^{2} \alpha}{27 E r}\left[-\left(2 s_{0}+1\right)\left(3 s_{0}^{2}-4 s_{0}-2\right) \theta+3\left(s_{0}+2\right)\right]\left(y^{2}-1\right) \\
& -2\left(\mu_{1}+\mu_{2}\right) K s_{0}^{2}\left(y^{2}-1\right), \\
& G=2 a \theta+4 a \theta s_{0}^{2}+6 a s_{0}-3 s_{0}-12 a s_{0}^{2}-16 a \theta s_{0}^{3}+10 a \theta s_{0}+6 a .
\end{aligned}
$$


Appendix E. The coefficients of the steady solutions for tangential anchoring in Poiseuille flows.

$$
\begin{aligned}
& H_{1}=\frac{6\left(s_{0}+2\right)\left(3+\theta\left(1-s_{0}\right)\right)}{4\left(\mu_{1} s_{0}+3 \eta\right)\left(s_{0}+2\right)\left(3+\theta\left(1-s_{0}\right)\right)+\alpha s_{0}\left(1-\lambda_{L}\right)\left(-a \theta\left(1-s_{0}\right)\left(s_{0}+2\right)+9 s_{0}\left(1-\lambda_{L}\right)\right)}, \\
& H_{2}=\frac{9\left(\lambda_{L}-1\right)}{4\left(\mu_{1} s_{0}+3 \eta\right)\left(s_{0}+2\right)\left(3+\theta\left(1-s_{0}\right)\right)+\alpha s_{0}\left(1-\lambda_{L}\right)\left(-a \theta\left(1-s_{0}\right)\left(s_{0}+2\right)+9 s_{0}\left(1-\lambda_{L}\right)\right)}, \\
& D=-C_{1}\left(9 H_{2}^{2} E_{2}+6 H_{2}^{2} F_{2}+2 G_{2} H_{1} H_{2}\right), \\
& E=-12 A_{1}\left(9 H_{2}^{2} E_{2}+6 H_{2}^{2} F_{2}+2 G_{2} H_{1} H_{2}\right)+C_{1}\left(6 H_{2}^{2} E_{2}+6 H_{2}^{2} F_{2}+2 G_{2} H_{1} H_{2}\right) \\
& +12 A_{2}\left(9 H_{2}^{2} E_{1}+6 H_{2}^{2} F_{1}+2 G_{2} H_{1} H_{2}\right) \text {, } \\
& F=2 A_{1}\left(6 H_{2}^{2} E_{2}+6 H_{2}^{2} F_{2}+2 G_{2} H_{1} H_{2}\right)-C_{1} H_{2}^{2} E_{2}+6 H_{2}^{2} E_{2} \\
& -2 A_{2}\left(6 H_{2}^{2} E_{1}+6 H_{2}^{2} F_{1}+2 G_{2} H_{1} H_{2}\right), \\
& R_{11}=-\frac{D}{C}, \quad R_{12}=-\frac{12 B R_{11}+E}{C}, \quad R_{13}=-\frac{24 A R_{11}+2 B R_{12}+F}{C}, \\
& K_{11}=\frac{1}{\left(\lambda^{2}-\mu^{2}\right)}\left[\frac{4 H_{2}^{2}\left(E_{2} A_{1}-E_{1} A_{2}\right)}{A}-12 . R_{11}-2 R_{12}+\left(R_{11}+R_{12}+R_{13}\right) \mu^{2}\right], \\
& K_{12}=\frac{1}{\left(-\lambda^{2}+\mu^{2}\right)}\left[\frac{4 H_{2}^{2}\left(E_{2} A_{1}-E_{1} A_{2}\right)}{A}-12 . R_{11}-2 R_{12}+\left(R_{11}+R_{12}+R_{13}\right) \lambda^{2}\right], \\
& K_{21}=-\frac{K_{11}\left(B_{2} \lambda^{2}+D_{2}\right)}{A_{2} \lambda^{2}}, \quad K_{22}=-\frac{K_{12}\left(B_{2} \mu^{2}+D_{2}\right)}{A_{2} \mu^{2}}, \\
& R_{21}=-\frac{D_{2} R_{12}-6 H_{2}^{2} E_{2}-6 H_{2}^{2} F_{2}-2 G_{2} H_{1} H_{2}}{12 A_{2}}, \quad R_{22}=-\frac{2 . B_{2} R_{12}+D_{2} R_{13}+E_{2} H_{2}^{2}}{2 . A_{2}}, \\
& N_{1}=\frac{G \alpha}{54 E r}\left(\frac{K_{21} \lambda^{2} \cosh (\lambda y)}{\cosh (\lambda)}+\frac{K_{22} \mu^{2} \cosh (\mu y)}{\cosh \mu}+12 R_{21} y^{2}+2 S_{22}\right) \\
& +\frac{a N s_{0}\left(1-4 s_{0}\right) \alpha}{3}\left[K_{21}\left(\frac{\cosh (\lambda y)}{\cosh (\lambda)}-1\right)+K_{22}\left(\frac{\cosh (\mu y)}{\cosh (\mu)}-1\right)+R_{21}\left(y^{4}-1\right)+S_{22}\left(y^{2}-1\right)\right] \\
& +\frac{\left(-4 a \theta+3 s_{0}+16 a \theta s_{0}^{3}-12 a+12 a s_{0}^{2}-12 a \theta s_{0}\right) \alpha}{108 E r}\left(\frac{K_{11} \lambda^{2} \cosh (\lambda y)}{\cosh (\lambda)}+\frac{K_{12} \mu^{2} \cosh (\mu y)}{\cosh (\mu)}\right. \\
& \left.+12 R_{11} y^{2}+2 R_{12}\right) \\
& +\frac{N s_{0}\left(1+2 s_{0}\right) a \alpha}{3}\left[K_{11}\left(\frac{\cosh (\lambda y)}{\cosh (\lambda)}-1\right)+K_{12}\left(\frac{\cosh (\mu y)}{\cosh (\mu)}-1\right)+R_{11}\left(y^{4}-1\right)+R_{12}\left(y^{2}-1\right)\right] \\
& +\frac{H_{2}{ }^{2} s_{0} \alpha}{27}\left[4 a\left(s_{0}-1\right)\left(2 s_{0}+1\right)\left(3 s_{0}+1\right) \theta+3\left(-2 a s_{0}+3 s_{0}+6 a s_{0}{ }^{2}-4 a\right)\right]\left(3 y^{2}-1\right)^{2} \\
& -\frac{2 a s_{0} \alpha}{9} H_{2}^{2}\left[\left(2 s_{0}+1\right)\left(3 s_{0}^{2}-5 s_{0}-4\right) \theta-6\left(s_{0}+2\right)\right] y^{2}\left(y^{2}-1\right)+4 \mu_{2} H_{1} H_{2} s_{0}^{2} y^{2}\left(y^{2}-1\right) \text {, } \\
& N_{2}=-\frac{G \alpha}{54 E r}\left(\frac{K_{21} \lambda^{2} \cosh \lambda y}{\cosh (\lambda)}+\frac{K_{22} \mu^{2} \cosh \mu y}{\cosh \mu}+12 R_{21} y^{2}+2 S_{22}\right) \\
& +\frac{a N s_{0}\left(-1+4 s_{0}\right) \alpha}{3}\left[K_{21}\left(\frac{\cosh (\lambda y)}{\cosh (\lambda)}-1\right)+K_{22}\left(\frac{\cosh (\mu y)}{\cosh (\mu)}-1\right)\right. \\
& \left.+R_{21}\left(y^{4}-1\right)+S_{22}\left(y^{2}-1\right)\right] \\
& +\frac{\left(8 a \theta+12 a-3+8 a s_{0}-16 a s_{0}^{2}-12 s_{0}\right) s_{0} \alpha}{108 E r}\left(\frac{K_{11} \lambda^{2} \cosh (\lambda y)}{\cosh (\lambda)}+\frac{K_{12} \mu^{2} \cosh (\mu y)}{\cosh (\mu)}+12 R_{11} y^{2}+2 R_{11}\right) \\
& +\frac{2 N s_{0}\left(1-s_{0}\right) a \alpha}{3}\left[K_{11}\left(\frac{\cosh (\lambda y)}{\cosh (\lambda)}-1\right)+K_{12}\left(\frac{\cosh (\mu y)}{\cosh (\mu)}-1\right)+R_{11}\left(y^{4}-1\right)+R_{11}\left(y^{2}-1\right)\right] \\
& +\frac{H_{2}^{2} s_{0} \alpha}{27 E r}\left[-2 a\left(s_{0}-1\right)\left(2 s_{0}+1\right)\left(6 s_{0}+1\right) \theta+3\left(4 a s_{0}-3 s_{0}-6 a s_{0}^{2}+2 a\right)\right]\left(3 y^{2}-1\right)^{2} \\
& -\frac{2 a s_{0} H_{2}{ }^{2} \alpha}{9 E r}\left[-\left(2 s_{0}+1\right)\left(3 s_{0}^{2}-4 s_{0}-2\right) \theta+3\left(s_{0}+2\right)\right] y^{2}\left(y^{2}-1\right) \\
& +4\left(\mu_{1}+\mu_{2}\right) H_{1} s_{0} y^{2}\left(y^{2}-1\right), \\
& G=2 a \theta+4 a \theta s_{0}^{2}+6 a s_{0}-3 s_{0}-12 a s_{0}^{2}-16 a \theta s_{0}^{3}+10 a \theta s_{0}+6 a .
\end{aligned}
$$


Appendix F. The coefficients of the steady solutions in normal anchoring in Poiseuille flows.

$$
\begin{aligned}
& H_{1}=\frac{6\left(s_{0}+2\right)\left(\theta\left(2 s_{0}+1\right)+3\right)}{4\left(\mu_{1} s_{0}+3 \eta\right)\left(s_{0}+2\right)\left(\theta\left(2 s_{0}+1\right)+3\right)+\alpha s_{0}\left(1+\lambda_{L}\right)\left(a \theta\left(s_{0}+2\right)\left(2 s_{0}+1\right)+9 s_{0}\left(1+\lambda_{L}\right)\right)}, \\
& H_{2}=\frac{9\left(1+\lambda_{L}\right)}{4\left(\mu_{1} s_{0}+3 \eta\right)\left(s_{0}+2\right)\left(\theta\left(2 s_{0}+1\right)+3\right)+\alpha s_{0}\left(1+\lambda_{L}\right)\left(a \theta\left(s_{0}+2\right)\left(2 s_{0}+1\right)+9 s_{0}\left(1+\lambda_{L}\right)\right)}, \\
& R_{1}=-\frac{2\left(9 H_{2}^{2} E_{1}+6 H_{2}^{2} F_{1}+2 G_{1} H_{1} H_{2}\right)+\left(9 H_{2}^{2} E_{2}+6 H_{2}^{2} F_{2}+2 G_{2} H_{1} H_{2}\right)}{2 D_{1}+D_{2}}, \\
& S_{1}=\frac{2\left(6 H_{2}^{2} E_{1}+6 H_{2}^{2} F_{1}+2 G_{1} H_{1} H_{2}\right)+\left(6 H_{2}^{2} E_{2}+6 H_{2}^{2} F_{2}+2 G_{2} H_{1} H_{2}\right)-12\left(2 B_{1}+B_{2}\right) R_{1}}{2 D_{1}+D_{2}}, \\
& T_{1}=-\frac{2 H_{2}^{2} E_{1}+2 H_{2}^{2} E_{2}+2\left(2 B_{1}+B_{2}\right) S_{1}}{2 D_{1}+D_{2}}, \quad K_{11}=-\left(R_{1}+S_{1}+T_{1}\right), \\
& K_{21}=-\frac{B_{1} \lambda^{2}+D_{1}}{A_{1} \lambda^{2}+C_{1}} K_{11}, \quad R_{2}=-\frac{D_{1} R_{1}+\left(9 H_{2}^{2} E_{1}+6 H_{2}^{2} F_{1}+2 G_{1} H_{1} H_{2}\right)}{C_{1}}, \\
& S_{2}=\frac{\left(6 H_{2}^{2} E_{1}+6 H_{2}^{2} F_{1}+2 G_{1} H_{1} H_{2}\right)-12 A_{1} R_{2}-12 R_{1} B_{1}-D_{1} S_{1}}{C_{1}}, \\
& T_{2}=\frac{H_{2}^{2} E_{1}+2 A_{1} S_{2}+2 S_{1} B_{1}+D_{1} T_{1}}{C_{1}}, \quad K_{22}=-\left(K_{21}+R_{2}+S_{2}+T_{2}\right), \\
& N_{1}=\frac{G \alpha}{54 E r}\left(\frac{K_{21} \lambda^{2} \cosh (\lambda y)}{\cosh (\lambda)}+\frac{K_{22} \mu^{2} \cosh (\mu y)}{\cosh \mu}+12 R_{2} y^{2}+2 S_{2}\right) \\
& +\frac{a N s_{0}\left(1-4 s_{0}\right) \alpha}{3}\left[K_{21}\left(\frac{\cosh (\lambda y)}{\cosh (\lambda)}-1\right)+K_{22}\left(\frac{\cosh (\mu y)}{\cosh (\mu)}-1\right)\right. \\
& \left.+R_{2}\left(y^{4}-1\right)+S_{2}\left(y^{2}-1\right)\right] \\
& +\frac{\left(-4 a \theta+3 s_{0}+16 a \theta s_{0}^{3}-12 a+12 a s_{0}^{2}-12 a \theta s_{0}\right) \alpha}{108 E r}\left(\frac{K_{11} \lambda^{2} \cosh (\lambda y)}{\cosh (\lambda)}+12 R_{1} y^{2}+2 S_{1}\right) \\
& +\frac{N s_{0}\left(1+2 s_{0}\right) a \alpha}{3}\left[K_{11}\left(\frac{\cosh (\lambda y)}{\cosh (\lambda)}-1\right)+R_{1}\left(y^{4}-1\right)+S_{1}\left(y^{2}-1\right)\right] \\
& +\frac{H_{2}^{2} s_{0} \alpha}{27}\left[4 a\left(s_{0}-1\right)\left(2 s_{0}+1\right)\left(3 s_{0}+1\right) \theta+3\left(-2 a s_{0}+3 s_{0}+6 a s_{0}{ }^{2}-4 a\right)\right]\left(3 y^{2}-1\right)^{2} \\
& -\frac{2 a s_{0} \alpha}{9} H_{2}^{2}\left[\left(2 s_{0}+1\right)\left(3 s_{0}^{2}-5 s_{0}-4\right) \theta-6\left(s_{0}+2\right)\right] y^{2}\left(y^{2}-1\right)+4 \mu_{2} H_{1} H_{2} s_{0}^{2} y^{2}\left(y^{2}-1\right), \\
& N_{2}=-\frac{G \alpha}{54 E r}\left(\frac{K_{21} \lambda^{2} \cosh \lambda y}{\cosh (\lambda)}+\frac{K_{22} \mu^{2} \cosh \mu y}{\cosh \mu}+12 R_{2} y^{2}+2 S_{2}\right) \\
& +\frac{a N s_{0}\left(-1+4 s_{0}\right) \alpha}{3}\left[K_{21}\left(\frac{\cosh (\lambda y)}{\cosh (\lambda)}-1\right)+K_{22}\left(\frac{\cosh (\mu y)}{\cosh (\mu)}-1\right)\right. \\
& \left.+R_{2}\left(y^{4}-1\right)+S_{2}\left(y^{2}-1\right)\right] \\
& +\frac{\left(8 a \theta+12 a-3+8 a s_{0}-16 a s_{0}^{2}-12 s_{0}\right) s_{0} \alpha}{108 E r}\left(\frac{K_{11} \lambda^{2} \cosh (\lambda y)}{\cosh (\lambda)}+12 R_{1} y^{2}+2 S_{1}\right) \\
& +\frac{2 N s_{0}\left(1-s_{0}\right) a \alpha}{3}\left[K_{11}\left(\frac{\cosh (\lambda y)}{\cosh (\lambda)}-1\right)+R_{1}\left(y^{4}-1\right)+S_{1}\left(y^{2}-1\right)\right] \\
& +\frac{H_{2}^{2} a s_{0} \alpha}{27 E r}\left[-2 a\left(s_{0}-1\right)\left(2 s_{0}+1\right)\left(6 s_{0}+1\right) \theta+3\left(4 a s_{0}-3 s_{0}-6 a s_{0}^{2}+2 a\right)\right]\left(3 y^{2}-1\right)^{2} \\
& -\frac{2 a s_{0} H_{2}^{2} \alpha}{9 E r}\left[-\left(2 s_{0}+1\right)\left(3 s_{0}^{2}-4 s_{0}-2\right) \theta+3\left(s_{0}+2\right)\right] y^{2}\left(y^{2}-1\right) \\
& +4\left(\mu_{1}+\mu_{2}\right) H_{1} s_{0} y^{2}\left(y^{2}-1\right) \\
& G=2 a \theta+4 a \theta s_{0}^{2}+6 a s_{0}-3 s_{0}-12 a s_{0}^{2}-16 a \theta s_{0}^{3}+10 a \theta s_{0}+6 a .
\end{aligned}
$$




\section{REFERENCES}

[1] W. R. Burghardt, Molecular orientation and rheology in sheared lyotropic liquid crystalline polymers, Macromol. Chem. Phys., 199 (1998), pp. 471-488.

[2] M. C. Calderer and B. Mukherjee, Chevron patterns in liquid crystal flows, Phys. D, 98 (1996), pp. 201-224.

[3] M. C. Calderer and B. Mukherjee, On Poiseuille flow of polymeric liquid crystals, Liq. Cryst., 22 (1997), pp. 121-136.

[4] T. CARLSSOn, Theoretical investigation of the shear flow of nematic liquid crystals with the Leslie viscosity $\alpha>0$ : Hydrodynamic analogue of first order phase transitions, Mol. Cryst. Liq. Cryst., 104 (1984), pp. 307-334.

[5] T. CARLsson, Unit-sphere description of nematic flows, Phys. Rev. A, 34 (1986), pp. 33933404.

[6] S. Chandrasekhar, Liquid Crystals, 2nd ed., Cambridge University Press, Cambridge, 1992.

[7] P. E. Cladis, S. Torza, in Colloid and Interface Science, vol. 4, M. Kerker, ed., Academic Press, New York, 1976.

[8] P. G. De Gennes and J. Prost, The Physics of Liquid Crystals, Oxford University Press, London, 1993.

[9] A. M. Donald and A. H. Windle, Liquid Crystalline Polymers, Cambridge Solid State Sci. Ser., Cambridge University Press, Cambridge, 1992.

[10] M. G. Forest AND Q. WANG, Monodomain response of finite-aspect-ratio macromolecules in shear and related linear flows, Rheol. Acta, 42 (2003), pp. 20-46.

[11] M. G. Forest, Q. WANG, And H. Zhou, Exact banded patterns from a Doi-MarrucciGreco model of nematic liquid crystal polymers, Phys. Rev. E, 61 (2000), pp. 66556662 .

[12] M. G. Forest, Q. WANG, AND H. ZhOU, Methods for the exact construction of mesoscale spatial structures in liquid crystal polymers, Phys. D, 152-153 (2001), pp. 288-309.

[13] M. G. Forest, R. Zhou, AND Q. WANG, Full-tensor alignment criteria for sheared nematic polymers, J. Rheol., 47 (2003), pp. 105-127.

[14] M. G. Forest, Q. WANG, H. Zhou, AND R. Zhou, Structure scaling properties of confined nematic polymers in plane Couette cells: The weak flow limit, J. Rheol., 48 (2004), pp. $175-192$.

[15] M. G. Forest, Q. WAng, And R. Zhou, The weak shear phase diagram for nematic polymers, Rheol. Acta, 43 (2004), pp. 17-37.

[16] M. G. Forest, Q. WAng, And R. Zhou, The flow-phase diagram of Doi-Hess theory for sheared nematic polymers II: Finite shear rates, Rheol. Acta, 44 (2004), pp. 80-93.

[17] M. G. Forest, Q. WANG, AND H. ZHOU, Structure formation in sheared tumbling nematic liquid crystal polymers, University of North Carolina, Chapel Hill, NC, preprint, 2004.

[18] M. G. Forest, R. Zhou, Q. Wang, X. Zheng, and R. Lipton, Anisotropy and heterogeneity of nematic polymer nano-composite film properties, in Modeling of Soft Matter, IMA Math. Appl., Vol. 141, M.-C. T. Calderer and E. M. Terentjev, eds., Springer-Verlag, New York, 2005, pp. 85-98.

[19] M. G. Forest, X. Zheng, R. Zhou, Q. Wang, and R. Lipton, Anisotropy and dynamic ranges in effective properties of of nematic polymer nano-composites. Adv. Func. Mat., 15 (2005), pp. 2029-2035.

[20] D. D. Joseph, Fluid Dynamics of Viscoelastic Liquids, Applied Math (N.Y.) 84, SpringerVerlag, New York, 1990.

[21] R. Kupferman, M. Kawaguchi, and M. M. Denn, Emergence of structure in a model of liquid crystalline polymers with elastic coupling, J. Non-Newtonian Fluid Mech., 91 (2000), pp. 255-271.

[22] R. G. Larson, The Structure and Rheology of Complex Fluids, Oxford University Press, London, 1999.

[23] R. G. LARSON, Roll-cell instabilities in shearing flows of nematic polymers, J. Rheol., 37 (1993), page 175.

[24] R. G. LaRson and D. W. Mead, Development of orientation and texture during shearing of liquid-crystalline polymers, Liq. Cryst., 12 (1993), pp. 751-768.

[25] R. G. LaRson And D. W. Mead, The Ericksen number and Deborah number cascade in sheared polymeric nematics, Liq. Cryst., 15 (1993), pp. 151-169.

[26] P. Manneville, The transition to turbulence in nematic liquid crystals: Part 1, general review. Part 2, on the transition via tumbling, Mol. Cryst. Liq. Cryst., 70 (1981), pp. 223-250. 
[27] G. MarruCCI, Tumbling regime of liquid-crystalline polymers, Macromol., 24 (1991), pp. $4176-4182$.

[28] G. Marrucci AND F. Greco, Flow behavior of liquid crystalline polymers, Adv. Chem. Phys., 86 (1993), pp. 331-404.

[29] A. D. Rey and M. M. Denn, Dynamical phenomena in liquid-crystalline materials, Annual Rev. Fluid Mech., 34 (2002), pp. 233-266.

[30] G. Sgalari, G. L. Leal, And J. Feng, The shear flow behavior of LCPs based on a generalized Doi model with distortional elasticity, J. Non-Newtonian Fluid Mech., 102 (2002), pp. 361-382.

[31] Z. TAN AND G. C. BERRY, Studies on the texture of nematic solutions of rodlike polymers, 3. Rheo-optical and rheological behavior in shear, J. Rheol., 47 (2003), pp. 73-104.

[32] T. Tsuji AND A. D. Rey, Effect of long range order on sheared liquid crystalline polymers, Part 1: Compatibility between tumbling behavior and fixed anchoring, J. NonNewtonian Fluid Mech., 73 (1997), pp. 127-152.

[33] Q. WAng, A hydrodynamic theory of nematic liquid crystalline polymers of different configurations, J. Chem. Phys., 116 (2002), pp. 9120-9136.

[34] X. Zheng, M. G. Forest, R. Lipton, R. Zhou, and Q. Wang, Exact scaling laws for electrical conductivity properties of nematic polymer nano-composite monodomains, Adv. Func. Mat., 15 (2005), pp. 627-638. 